\title{
Phase Transition in the 3d Random Field Ising Model
}

\author{
J. Bricmont ${ }^{\star}$ and A. Kupiainen $\star \star$ \\ Institute for Theoretical Physics, University of California, Santa Barbara, California 93106, USA
}

\begin{abstract}
We show that the three-dimensional Ising model coupled to a small random magnetic field is ordered at low temperatures. This means that the lower critical dimension, $d_{l}$ for the theory is $d_{l} \leqq 2$, settling a long controversy on the subject. Our proof is based on an exact Renormalization Group (RG) analysis of the system. This analysis is carried out in the domain wall representation of the system and it is inspired by the scaling arguments of Imry and Ma. The RG acts in the space of Ising models and in the space of random field distributions, driving the former to zero temperature and the latter to zero variance.
\end{abstract}

\section{Introduction}

An interesting class of disordered systems is obtained by coupling impurities to the order parameter of a statistical system. This situation may be modelled e.g., by the Ising model (or the $\phi^{4}$-theory) with a random magnetic field. This random field Ising model (RFIM) describes actual physical systems, such as dilute antiferromagnets in a uniform field [1] and has been used to study, among other things, the effects of impurities on the fluctuations of interfaces [2,3]. It has also served as a useful playground for various theoretical ideas: the replica trick, $[4,5]$ dimensional reduction $[6,7,8,9]$ and supersymmetry $[10]$.

As usual, one of the interesting theoretical questions is to determine the upper and lower critical dimensions of the model. The most elegant argument for the upper critical dimension $d_{u}$ (i.e., the dimension above which the theory is Gaussian in the infrared) is dimensional reduction, which says that, at long distances and near $d_{u}$, the (quenched) correlation functions for the random system behave as those of the corresponding deterministic system, but in two less dimensions. The argument was, basically, to replace the random system by its tree approximation, a stochastic differential (or difference) equation, which, via its representation in

* Permanent address: Physique Theorique, U.C.L., 2 ch. du Cyclotron, B-1348 Louvain-la-Neuve, Belgium

$\star \star$ Permanent address: Research Institute for Theoretical Physics, Helsinki University, SF-00170 Helsinki, Finland 
terms of a SUSY field theory was argued (and proved [11]) to undergo dimensional reduction. While this argument is now known to have many problems, $[12,13]$, its prediction, $d_{u}=6$, is nevertheless believed to be correct.

Since the lower critical dimension $d_{l}$ (above which there is symmetry breaking) of the deterministic system is one, the formal extension $[4,16]$ of the dimensional reduction argument would yield $d_{l}=3$ for the random system. This result was in contradiction with the earlier scaling arguments of Imry and Ma, [14] which predicted $d_{l}=2$. The controversy was amplified further by the study of the SOS and related interface models for the RFIM. The interface between + and - spins in a system of linear size $L$ was found to diverge as $L^{(5-d) / 2}$ by the use of the replica trick [4]. This was consistent with the dimensional reduction result, $d_{l}=3$, because, if an interface diverges like its linear size $L$, one does not expect separation of the system into + and - phases. On the other hand, an Imry-Ma scaling argument $[15,2]$, yielded $L^{(5-d) / 3}$, predicting $d_{l}=2$. The situation was subsequently greatly clarified by two results. First, Fisher, Fröhlich and Spencer [16] and Chalker [7] put the Imry-Ma argument on a much more solid basis and finally a strong case for $d_{l}=2$ was made by Imbrie [18], who proved rigorously, that at zero temperature, the ground state in $d=3$ is ordered. However, the case for $d_{l}=3$ could still be made (on the basis of dimensional reduction): the same result for the ground state also holds for the pure system at $d=1$, but for $T \neq 0$ the onedimensional Ising model is disordered.

In this paper we settle the controversy by proving that, for $d=3$ and small disorder, the RFIM is ordered at low temperatures. Thus $d_{l} \leqq 2$.

Although our result is at variance with the above mentioned predictions of some $\varepsilon$ expansions (with $\varepsilon=5-d$ ), our proof vindicates rather than contradicts the Renormalization Group approach to phase transitions. Indeed, we construct, with no replicas or dimensional interpolations, a straightforward Renormalization Group transformation (suggested by the Imry-Ma argument) under which the system flows towards the zero disorder, zero temperature trivial fixed point.

A combination of our result with the one of $[19,20]$ on the large disorder regime shows that a phase transition occurs when the variance of the disorder is varied (for $d \geqq 3$ and at low temperatures). Moreover we show that, in the ordered phases, the correlation functions cluster exponentially with probability one.

An extension of our method should prove the following results for interfaces, again at low temperatures and small disorder: for $d \geqq 4$, the interface is essentially flat (like in the deterministic model for $d \geqq 3$ ) and for $d=3$, the divergence is bounded from above by $L^{2 / 3}\left(=L^{(5-d) / 3}\right)$.

We do not, at present, completely prove the conjecture $d_{l}=2$, because we are unable to prove that, in $d=2$, an arbitrary small disorder destroys the phase transition, as is suggested by the Imry-Ma argument. However, we have constructed, and explicitly solved, a hierarchical random field model where our Renormalization Group transformation becomes exact [21]. This model has the following properties: for $d \geqq 3$, the system is ordered while, in $d=2$, there is no spontaneous magnetization but the correlation functions have a power law decay (such a decay was also found in $d=2$ RFIM, at zero temperature [22]). However, we believe that this power law decay is an artifact of the hierarchical model. 
Let us now discuss the main ideas of our proof (a sketch of which is in [23] and is outlined in more technical terms in Sect. 3). It is based on the Imry-Ma scaling argument combined with a Renormalization Group approach to first order phase transitions due to [24].

Why should one use the Renormalization Group here, since we are in a non-critical regime? The reason is that the randomness of the field induces, even in an ordered phase, large regions with the reverse magnetization. Since these (rare) events occur on all scales, a Renormalization Group analysis is natural.

Consider now what a Renormalization Group transformation (RGT) does: it maps, by integrating out some degrees of freedom, a class of (generalized) Ising or $\phi^{4}$ models into itself. One has then to see how the parameters of the model flow. Hopefully, only few parameters are relevant or marginal. One has to keep track of those precisely, while some gross information is sufficient for the many irrelevant parameters.

Since we are studying low temperatures, it is convenient to use the representation of the Ising model in terms of Peierls' contours. Thus, we map contour models into contour models and we do that for every realization of the fields. This induces a map on the random fields themselves so that the novel feature of our approach is that we study two flows: a deterministic one, acting on contours, and one acting on the probability distribution of the field. As we shall see, all the variables are irrelevant (in particular, the temperature) but we have to deal with the fact that the random field itself may be relevant from a deterministic point of view while it is irrelevant in probability.

We now describe our RGT: write the Ising partition function as

$$
Z=\sum_{\{\gamma\}} \prod_{\gamma} \rho(\gamma) \exp \left(\beta \sum_{x \in V^{-}} H_{x}\right),
$$

where the sum is over (compatible) Ising contours, $\rho(\gamma)=e^{-2 \beta|\gamma|}, H_{x}=-2 h_{x}$ and $V^{-}$is the region, determined by $\{\gamma\}$, where $\sigma_{x}=-1$. A more precise version of (1.1) is derived in Sect. 3.

Since we want to prove long range order (an infrared problem) the RG strategy consists in integrating out the short range fluctuations. These consist of the small contours and of the small wiggles in the large ones. We "sum out," by means of a convergent expansion, the small contours, (of diameter $\leqq L, L$ being a fixed number), thereby producing a new (effective) field. This is not completely local, since it has exponentially decaying tails. The latter are irrelevant and will remain approximately local under the RGT. The next step is the "blocking": we cover the lattice with disjoint $L$-cubes and resum all the large contours that intersect a given set of $L$-cubes (this eliminates the wiggles). The local part of the new effective field will be given by

$$
H_{x}^{\prime}=L^{-2} \sum_{y \in L x} H_{y}+\text { nonlinear, local, }
$$

where $L x$ is the $L$-cube centered at $x \in Z^{3}$ and the second term includes the local, non-linear part of new field produced by the summation of the small contours. It is so small that it will not affect the conclusion drawn from the linear analysis, based on the first term. 
Now, we rescale everything on $\mathbf{Z}^{3}$ and see how the parameters evolve:

$$
\rho(\gamma) \rightarrow \rho^{\prime}\left(\gamma^{\prime}\right) \simeq e^{-\beta^{\prime}\left|\gamma^{\prime}\right|}
$$

with $\beta^{\prime}=L^{2} \beta$ since $\left|\gamma^{\prime}\right|$ counts the number of $L$ cubes in $\gamma^{\prime}$ and contours scale like surfaces (at least those that have an interior, after blocking. Some do not and scale linearly; this forces us to introduce a new coupling, $\widetilde{\beta}$ which flows as $\widetilde{\beta}^{\prime}=L \widetilde{\beta}$ ). The scaling of $\beta$ explains the $L^{-2}$ factor in (1.2). From the linear part of (1.2), we see that $\left|H^{\prime}\right|$ may be as large as $L|H|$ (in which case it is relevant). However, probabilistically, it is irrelevant, having a variance $\varepsilon^{\prime 2}=L^{-1} \varepsilon^{2}$.

Moreover, since we have only local contributions in (1.2), the new random fields will remain almost independent of each other.

The conclusion is that, upon iteration, the system flows to the zero temperature, zero field fixed point. The main problem, as with all RG analysis, is the rigorous treatment of the (rare) large fields, which may appear on all scales.

The main observation, which allows us to handle the latter, is that a field may create contours around itself (if it points in the direction opposite to the magnetization) but only up to a length scale, depending on its strength. Moreover, the larger the field, the more unprobable it is. Thus, with large probability, a large field becomes small (in the sense that it does not anymore create contours) after a few iterations. However, we do not have convergent expansions inside the large field regions, which is another way of saying that Griffiths' singularities [25] may be present. This fact can create small denominator problems, which we avoid by carrying inductively suitable lower bounds on the part of the partition function localized at and nearby the large fields.

In Sect. 2 we state our results. In Sect. 3, after introducing our contour representation, we give an outline of the proof. The latter is divided into three parts: Sects. 4,5 and 6 are deterministic i.e., we apply a sequence of RGT to the partition function for every field configuration. Section 7 contains the probabilistic estimates and in Sect. 8 we use and slightly extend the previous work to control the correlation functions and finish the proofs.

\section{Results}

We consider the model given by the Hamiltonian

$$
-\mathscr{H}=\sum_{\langle x y\rangle}\left(\sigma_{x} \sigma_{y}-1\right)+\sum_{x} h_{x} \sigma_{x}
$$

where $\sigma_{x}= \pm 1, x \in \mathbf{Z}^{d}$ and $h_{x}$ are independent, Gaussian, random variables.

For every realization of the fields $\left\{h_{x}\right\}$, the infinite volume limit of the Gibbs states with + and - boundary conditions exist, by FKG inequalities. We denote by \langle\rangle$^{+},\langle\rangle^{-}$the expectation with respect to these infinite volume Gibbs states. Unless otherwise stated, we write $P$ for the probability distribution on the $h_{x}$ :

$$
P=\prod_{x \in \mathbf{Z}^{d}} \exp \left(-h_{x}^{2} / 2 \varepsilon^{2}\right) \frac{d h_{x}}{\sqrt{2 \pi} \varepsilon},
$$

and $\bar{f}$ for the average of $f$ with respect to (2.2). We shall write Pr to denote, when 
needed, the probability corresponding to a Gibbs state (for a given realization of $\left.\left\{h_{x} \mid x \in \mathbf{Z}^{d}\right\}\right)$.

2.1 Theorem. For $d \geqq 3, \beta$ large and $\varepsilon$ small enough,

$$
\left\langle\sigma_{0}\right\rangle^{+} \geqq 1-\exp (-0(\beta)),
$$

with probability at least $1-\exp \left(-0\left(\varepsilon^{-2}\right)\right)$. Therefore,

$$
\overline{\left\langle\sigma_{0}\right\rangle^{+}} \geqq 1-\exp (-0(\beta))-\exp \left(-0\left(\varepsilon^{-2}\right)\right) .
$$

And, by ergodicity (of $P$ )

$$
\lim _{V \rightarrow \infty}|V|^{-1} \sum_{x \in V}\left\langle\sigma_{x}\right\rangle^{+}=\overline{\left\langle\sigma_{0}\right\rangle^{+}} .
$$

Moreover, the correlation functions decay exponentially:

$$
\left|\left\langle\sigma_{A} \sigma_{B}\right\rangle^{+}-\left\langle\sigma_{A}\right\rangle^{+}\left\langle\sigma_{B}\right\rangle^{+}\right| \leqq c(h,|A|,|B|) \exp (-m d(A, B)),
$$

where $m=0(\beta)$ and $c(h,|A|,|B|)<\infty$ with probability one.

\subsection{Remarks.}

1. Our results extend, with no changes in the proofs, to any distribution of independent $h$ 's which is even and satisfies:

$$
\overline{\exp t h_{x}} \leqq \exp \left(0\left(\varepsilon^{2}\right) t^{2}\right), \quad t \in \mathbf{R} .
$$

Moreover, the interaction need not be nearest-neighbor. All we need is the contour representation and the symmetry between the + and the - phases at $h=0$.

2. The free energy and the correlation functions are $C^{\infty}$ in $\beta$ and (one-sided) $C^{\infty}$ in a uniform field $h$, at $h=0$. (This also follows from (2.6).) Analyticity is not expected to hold, because of Griffiths' singularities [25].

\section{Main Ideas of the Proof}

Here we set up some of our notation, define our contours and outline the rest of the proof.

3.1 Notations and Contour Representation. To define contours, start with the Ising partition function:

$$
Z^{+}(V)=\sum_{\sigma} \exp \left(-\beta \mathscr{H}^{+}(\sigma)\right)
$$

in a volume $V$ with + boundary conditions (which are implicit in $\mathscr{H}^{+}$). The sum is over all configurations equal to +1 in $V^{c}$. It is well known that there is in a one-to-one correspondence between configurations $\sigma$ and families of non-overlapping contours. For later purposes, we shall define contours in a slightly more general way, (which will be extended again later): A contour $\gamma=(\gamma, \sigma(\gamma))$ consists of its (connected) support $\gamma$ and $\sigma(\gamma)$, a configuration (on $\mathbf{Z}^{3}$ ) of signs which is constant on connected components of $\mathbf{Z}^{3} \backslash \underline{\gamma}$. A set is connected if the graph obtained by joining points $x, y$ with $d(x, y) \leqq 1$ is connected. Our metric is 
$|x|=\max _{i} x_{i}$. In the usual Ising contours $\sigma(\gamma)$ differs from one connected component to the other, but we shall use contours where $\sigma(\gamma)$ may be constant everywhere.

Each $\gamma$ partitions $\mathbf{Z}^{3}$ into the regions of + and - spins, i.e., according to the sign of $\sigma(\gamma)$, into subsets $V^{+}(\gamma), V^{-}(\gamma)$.

A family $\Gamma=\{\gamma\}$ of contours is compatible if the supports are pairwise disjoint and if the signs match, in which case $V^{+}(\Gamma), V^{-}(\Gamma)$ are unambiguously defined.

Before rewriting $Z^{+}(V)$ in terms of contours, we shall include, mostly for convenience, the region where the random fields are large into the contours, so we first define the latter. Let $\tilde{H}_{x} \equiv-2 h_{x}$. A field is small if $\left|\tilde{H}_{x}\right|<\delta$, where $\delta$ is a number which we shall choose small later. The small field is

$$
H_{x}=\tilde{H}_{x} \chi\left(\left|\tilde{H}_{x}\right| \leqq \delta\right)
$$

(this is defined everywhere, being zero if the field is large). For the large fields we introduce a discretization:

$$
\left.\left.n_{x}=\sum_{n=1}^{\infty}(n+1) \chi\left(\left|\tilde{H}_{x}\right| \in\right] n, n+1\right] \delta\right)
$$

so that

$$
\left|\tilde{H}_{x}\right| \leqq n_{x} \delta
$$

Later we shall introduce $N$ variables which, in the first stage, coincide with $n$ :

$$
N_{x}=n_{x} .
$$

The large field region is:

$$
D=\left\{x \mid N_{x}=n_{x} \neq 0\right\}=\left\{x|| \tilde{H}_{x} \mid>\delta\right\} .
$$

We write

where

$$
\begin{aligned}
Z^{+}(V) & =\exp (-\beta(H, V) / 2) Z, \\
Z & =\sum_{\underline{\Gamma} \supset D} \rho(\Gamma) \exp \left(\beta\left(H, V^{-}(\Gamma)\right)\right),
\end{aligned}
$$

$$
(f, V) \equiv \sum_{x \in V} f_{x}
$$

and, in (3.7), we pulled out the energy of the $(\varepsilon=0)$ ground state but only outside $D$. The constraint $\underline{\Gamma} \supset D$ means that the supports of the contours in $\Gamma$ must contain $D$. For example, $\sigma_{x}=+1$ everywhere corresponds to $\underline{\Gamma}=D$,

$$
\rho(\Gamma)=\exp (\beta(h, D)),
$$

and the contours are the connected components of $D$. In general,

where

$$
\rho(\Gamma)=\prod_{\gamma \in \Gamma} \rho(\gamma)
$$

$$
\rho(\gamma)=\exp \left(-2 \beta\left|\partial V^{-}(\gamma)\right|+\beta(h \sigma(\gamma), \underline{\gamma} \cap D)\right),
$$

if $\gamma$ corresponds to usual Ising contour, i.e. if $\underline{\gamma}$ is a connected set made of Ising contours and (parts of) $D$, and $\sigma(\gamma)$ is as in the Ising model; $\rho(\gamma)=0$ otherwise. In later stages, $\rho(\gamma)$ will not vanish for more general $\gamma$ 's. 
We shall only use the fact that $\rho(\gamma)$ are random variables such that

$$
\begin{aligned}
& \text { i) } \rho(\gamma) \text { depends on }\left\{h_{x} \mid x \in \underline{\gamma}\right\} \text {, } \\
& \text { ii) } \rho(\gamma)(h)=\rho(-\gamma)(-h) \text {, where }-\gamma \equiv(\underline{\gamma},-\sigma(\gamma)) \text {, } \\
& \text { iii) } 0 \leqq \rho(\gamma) \leqq \exp \left(-\beta\left|\partial V^{-}(\gamma)\right|-\tilde{\beta}|\underline{\gamma}|+\beta(N, D)\right) \text {, }
\end{aligned}
$$

where

$$
\partial V=\left\{\langle x y\rangle \mid x \in V, y \in V^{c}\right\}
$$

(Note that $\partial V^{-}=\partial V^{+}$.)

$$
\begin{gathered}
\tilde{\beta}=\beta^{1 / 2}, \\
|\underline{\gamma}|=\# \text { sites in } \underline{\gamma} .
\end{gathered}
$$

i) and ii) are easy to check. We write the rather cumbersome bound (iii) because $\beta$ and $\tilde{\beta}$ will flow differently under the RGT.

On the other hand, if $\gamma=a$ connected component of $D, \sigma(\gamma)=$ constant, one has the lower bound

$$
\rho(\gamma) \geqq \exp (-\beta(N, \underline{\gamma})) \text {. }
$$

3.2 Outline of the Proof. In Sect. 4, we do the first RGT. We start by summing out the small contours, defined by having a small diameter and by not touching or enclosing a large field. This, by means of a straightforward expansion, produces new fields, which we separate into local and non-local parts.

We decompose again the local part into small and large fields. Next, we block the fields and the large contours on a coarser lattice $L Z^{3}$ so that everything can be rescaled back to $\mathbf{Z}^{3}$ and the transformation can be iterated. At this point we face a problem: we want that the weights of the new, blocked, contours remain local in the sense that they depend, as random variables, on the fields near their support (like in (3.13)). Otherwise, the new effective fields obtained by summing out the small contours on the next scale would become uncontrollably non-local. And our probabilistic estimates depend very much on the fact that the new fields, given by (1.2), are local and thus remain almost independent.

Unfortunately, the locality is lost by summing out small contours because this generates interactions between the remaining large contours. Of course, one may, as we do, Mayer-expand these interactions and define new "contours" by putting together previous ones and Mayer graphs joining them. This restores locality (the Mayer graphs are just chains of polymers coming from previous steps) but, in general, may destroy the positivity of the contour weights. We need this positivity because, later, we shall use lower bounds on the partition functions near the large fields. The solution we adopt is, since the interactions are uniformly bounded, to add and subtract their minimal value, and therefore, deal only with positive Mayer graphs.

Another point to stress is that we want our new field to be random variables defined for every (original) field configuration. This is used in the probabilistic estimates, because then we may estimate the size of the new field by means of the original field distribution, which is simple (independent random variables). Otherwise, one would have to condition on events that would make the new fields 
well defined. However, this goal is achieved simply by defining the new fields to be zero whenever the previous large fields might make them ill-defined.

In Sect. 5, we show that the partition function produced in Sect. 4 is stable under the RGT. The weights of the contours remain local and satisfy (3.15) with $\beta, \widetilde{\beta}$ flowing as:

$$
\beta_{k}=L^{(2-\alpha) k} \beta, \quad \widetilde{\beta}_{k}=L^{(1-\alpha) k} \tilde{\beta},
$$

because $\beta_{k}$ multiplies, in (3.15), $\left|\partial V^{-}(\gamma)\right|$, which scales like a surface while $\tilde{\beta}_{k}$ multiplies $|\gamma|$ which may be one-dimensional (some contours have $\sigma(\gamma)=$ const, hence $\partial V^{-}=\emptyset$ ). $L^{-\alpha}$ is included because we do not have exact scaling i.e., we shall have to absorb some geometrical constants. Thus $\alpha$ can be taken as small one wants, provided $L$ is large enough.

The scaling of $\beta_{k}$ explains the scaling dimension of $H$, which is as in (1.2), with $L^{2-\alpha}$ replacing $L^{2}$ because we always control the field inside a small contour by $\beta\left|\partial V^{-}(\gamma)\right|$ and we want the same $\beta$ in front of both competing terms (if a contour has $\partial V^{-}(\gamma)=\emptyset$, there is no field to control).

The main new point in Sect. 5 is the absorption, into the small fields, of the large ones that have become small. This is done when the fields, under the iteration of (1.2), are of order $L^{-k} \varepsilon^{2}$. Then, $L^{-k} \varepsilon^{2} \beta_{k} \ll \widetilde{\beta}_{k}$, and we can control these (previously large) fields with $\widetilde{\beta}_{k}$ alone (we need that, because not all contours have a $\partial V^{-}(\gamma)$ ). This scale reflects the one on which a large field cannot create a contour anymore. This means that, if a contour becomes small on the $k^{\text {th }}$ step, then, on the original scale, $|\gamma| \geqq L^{k}$. If the $k^{\text {th }}$ step field inside this contour is small, then the total original field inside $\gamma,|h| \leqq L^{(2-\alpha) k} L^{-k} \varepsilon^{2}$, (by (1.2) the field decreases by $L^{-2+\alpha}$ at each step). Therefore $|h|$ was much less than $|\underline{\gamma}|$ to start with, and the contour was unlikely to be created by the field. The reason why this works is because a large field, equal, say, to $L^{k}$ on the first scale, will become small on a scale $L^{k_{0}}$ with $k_{0} \simeq 0(k)$ but has probability $\exp \left(-L^{2 k} / \varepsilon^{2}\right)$. Therefore the typical distance between such events $\left(=\exp 0\left(L^{2 k} / \varepsilon^{2}\right)\right)$ is very large compared to the scale $L^{O(k)}$ at which they "disappear."

The absorption of the large field is realized by pulling out of the partition function their contribution (provided that they are sufficiently far from the remaining large field). This would create serious small denominator problems if we did not have good lower bounds (like (3.19)) on these large fields contributions. These, and the upper bound (3.15) on the contour weights $\rho(\gamma)$ needed to iterate the RGT., are proven in Sect. 6. The proof is a tedious but straightforward consequence of our inductive definitions (and, for the lower bound, of the positivity of the contour weights). We also provide, in Sect. 6.3 some details on the convergence of our small contour expansions.

In Sect. 7 we prove our main probabilistic estimate: for any fixed site, the probability that there is a large field, on any scale, at that site, is small. This involves two arguments, because a "large field" can occur for two reasons: either because the new field is too large or by blocking the previous large fields. The probability distribution of the new field satisfies a Gaussian bound with a running variance

$$
\varepsilon_{k}^{-2}=L^{(1-\eta) k} \varepsilon^{-2}
$$

because, see (1.2), the sum of the fields in a volume $L^{3}$ scales like $L^{3 / 2}$ (and we 
divide by $L^{-2+\alpha}$ ). We should take $\eta=2 \alpha$, but we lose a little bit in our estimates and any $\eta>3 \alpha$ will do (for $L$ large).

Finally, we summarize the relations between the various constants appearing in the proof: fix $\alpha$ small, $\eta>3 \alpha$, take $L$ large so that $L^{-\alpha}, L^{-\eta}$ control various constants and let $\delta$ satisfy $\delta L^{3} \ll 1$ (so that the small fields inside the small contours are controlled by $\left.\left|\partial V^{-}(\gamma)\right|\right)$. Then we need $\beta$ and $\varepsilon^{-2}$ to be so large that the first small contour expansion converges $\left(\beta \gg L^{2}\right.$ will do) and $\delta^{2} \varepsilon^{-2} \gg 1$ so that, in Sect. 7, various probabilities are small. Moreover, we shall always proceed as if $\varepsilon^{2}$ is not too small, e.g., we write $e^{-c \tilde{\beta}} \leqq \varepsilon^{2}$ or $\beta \varepsilon^{2} \geqq 1$. From (3.20), (3.21) we see that these relations hold on all scales if they hold on the first one. And there we can simply replace, in our bounds, if necessary, the true $\varepsilon^{2}$ by some larger one (which is still sufficiently small).

\section{The Renormalization Group in the Contour Representation}

The RGT consists of two steps. In the first one, which we call "coarse graining," we integrate out the small scale fluctuations of the system. The second one, the "blocking," scales the distances so that the sum in the new partition function contains again contours on all scales. We discuss first the coarse graining operation.

4.1 Coarse Graining. We start with the Ising partition function (3.8),

$$
Z=\sum_{\Gamma \supset D} \rho(\Gamma) e^{\beta\left(H, V^{-}(\Gamma)\right)}
$$

with $\Gamma=\{\gamma\}$ a compatible set of contours $\gamma=(\underline{\gamma}, \sigma(\gamma))$ as explained before. Equation (4.1) is taken in a volume $V_{N} \equiv V$ and we produce from it a "renormalized" one, $Z^{\prime}$, in the volume $V_{N-1} \equiv V^{\prime}$. The idea is to fix in (4.1) "large" contours of size $>L$ and "sum out" all small contours, of size $\leqq L$, compatible with the large ones. Since the small ones can effectively be summed only when the field enclosed by them is not too big, we will call a contour $\gamma$ small if

$$
d(\underline{\gamma}) \leqq L \quad \text { and } \quad V(\gamma) \cap D=\emptyset,
$$

where $d(\underline{\gamma})$ is the diameter of the set $\underline{\gamma}(V(\gamma)=\underline{\gamma} \cup \operatorname{Int} \gamma$, where Int $\gamma$ is the set enclosed by $\underline{\gamma})-\gamma$ is large otherwise. Divide (4.1) into a sum $\sum^{l}$ over large contours and $\sum^{s}$ over the small ones. Then we may write

$$
Z=\sum_{\Gamma \supset D}^{l} \rho(\Gamma) e^{\beta\left(H, V^{-}\right)} Z_{s}^{+}\left(V^{+} \backslash \underline{\Gamma}\right) Z_{s}^{-}\left(V^{-} \backslash \underline{\Gamma}\right)
$$

with $V^{ \pm}=V^{ \pm}(\Gamma)$ and for any $\Lambda \subset D^{c}$,

$$
Z_{s}^{ \pm}(\Lambda)=\sum_{\Gamma}^{s} \rho(\Gamma) e^{ \pm \beta\left(H, V^{\mp}(\Gamma)\right)}
$$

are the small contour partition functions. To get (4.3), just write in (4.1) $\Gamma=\Gamma_{s} \cup \Gamma_{l}$ and

$$
\left(H, V^{-}(\Gamma)\right)=\left(H, V^{-}\left(\Gamma_{l}\right)\right)+\left(H, V^{-}\left(\Gamma_{s}\right) \cap V^{+}\left(\Gamma_{l}\right)\right)-\left(H, V^{+}\left(\Gamma_{s}\right) \cap V^{-}\left(\Gamma_{l}\right)\right) .
$$

Equation (4.4) may be analyzed by a standard convergent polymer expansion since it involves only contours of small diameter with small field within. We get (see 
Sect. 6.3)

$$
\frac{1}{\beta} \log Z_{s}^{ \pm}(\Lambda)=\sum_{C \subset \Lambda} \delta h_{C}^{ \pm} \equiv\left(\delta h^{ \pm}, \Lambda\right),
$$

where we extended our notation (3.9) to function on subsets of $\mathbf{Z}^{3} . \delta h_{C}^{ \pm}=0$ unless $C$ is connected, and $\delta h_{\mathrm{C}}^{ \pm}$are random variables satisfying

$$
\begin{aligned}
& \text { (i) } \delta h_{C}^{ \pm} \text {depends on } h \mid C, \\
& \text { (ii) } \delta h_{C}^{+}(h)=\delta h_{C}^{-}(-h), \\
& \text { (iii) }\left|\delta h_{C}^{ \pm}\right| \leqq e^{-\tilde{\beta}|C| / 2}, \quad \tilde{\beta}=\beta^{1 / 2} .
\end{aligned}
$$

To have $\delta h^{ \pm}$everywhere defined, we set

$$
\delta h_{C}^{ \pm}=0 \text { for } C \cap D \neq \emptyset \text { or } C \cap V^{c} \neq \emptyset .
$$

$\delta h_{\bar{C}}^{ \pm}$are non-local contributions to the effective random field obtained after coarse graining. Inserting (4.5) into (4.3), we have

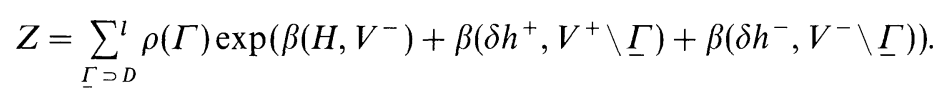

We will finish the coarse graining by pulling out of $Z$ the + state contribution [remember (4.9)]:

$$
\begin{aligned}
Z & =\exp \left[\beta\left(\delta h^{+}, V\right)\right] Z^{\prime} \\
Z^{\prime} & =\sum_{\Gamma \supset D}^{l} \rho(\Gamma) \exp \left[\beta\left(H+\chi_{\Gamma}^{c} \delta H, V^{-}\right)-\beta\left(\chi_{\Gamma} \delta h^{+}, V\right)\right],
\end{aligned}
$$

where

$$
\delta H=\delta h^{-}-\delta h^{+}
$$

and, since $\delta h^{ \pm}$in (4.10) are only on $\Gamma^{c}$, we introduced the functions

$$
\chi_{\Gamma}(C)= \begin{cases}1 & \text { if } \Gamma \cap C \neq \emptyset \\ 0 & \text { otherwise }\end{cases}
$$

with $\chi_{\Gamma}^{c}=1-\chi_{\Gamma}$. Finally, put $\delta H$ everywhere on $V^{-}$:

$$
Z^{\prime}=\sum_{I \supset D}^{l} \rho(\Gamma) e^{\beta\left(H+\delta H, V^{-}\right)} \exp \left[-\beta\left(\chi_{\Gamma} \delta h^{+}, V\right)-\beta\left(\chi_{\Gamma} \delta H, V^{-}\right)\right] .
$$

This is the result of the coarse graining. It produced small non-local contributions to $H$ and a weak interaction between the contours (the last two terms).

4.2 Blocking. Equation (4.14') involves only large contours (and possibly small ones in $D$ ), but they may have small scale fluctuations in their shape. The blocking consists of a grouping of the contours and other terms in $Z^{\prime}$ into sets defined on a coarser lattice $L Z^{3}$ followed by an appropriate rescaling back to the unit lattice $\mathbf{Z}^{3}$. Thus, given a $y \in \mathbf{Z}^{3}$, we may write $y=L x+z$ with $x \in \mathbf{Z}^{3}$ and $\left|z_{\mu}\right|<L / 2$, $\mu=1,2,3$ (remember that $L$ is odd). We define

$$
\left[L^{-1} y\right]=x \text {. }
$$


If no confusion arises, we denote the block of $y$ 's satisfying (4.15) by $L x$. Also, for a set $C,[C / L]=\{[y / L] \mid y \in C\}$ and $L C=\{L x \mid x \in C\}$. We start by blocking the field $H$. The local part of the blocked field is defined to be

$$
\tilde{H}_{x}^{\prime}=L^{-2+\alpha} \sum(H+\delta H)_{C} / n(C)
$$

with the constraint $C \subset L x$, or $C \cap L x \neq \emptyset, d(C) \leqq L / 4$. This involves an average of $(H+\delta H)_{y}$ in $L x$ together with $C$ 's reaching out to $L y$ with $|y-x|<2$ in the new lattice. $n(C)=|\{x \mid C \cap L x \neq \emptyset\}|$. The nonlocal part of $H^{\prime}$ is

$$
\tilde{H}_{C^{\prime}}^{\prime}=L^{-2+\alpha} \sum_{\left[L^{-1} C\right]=C^{\prime}} \delta H_{C} \quad\left|C^{\prime}\right| \geqq 2
$$

with $d(C)>L / 4$ in the sum. These definitions give e.g.,

$$
\beta(H+\delta H, V)=\beta^{\prime}\left(\tilde{H}^{\prime},\left[L^{-1} V\right]\right)
$$

(use (4.9) again), with

$$
\beta^{\prime}=L^{2-\alpha} \beta
$$

Clearly we have

(i) $\tilde{H}_{C^{\prime}}^{\prime}$ depends on $\left.h\right|_{L C^{\prime}}$ and is odd as a function of $h$,

(ii) $\tilde{H}_{x}^{\prime}$ and $\tilde{H}_{y}^{\prime}$ are independent for $|x-y| \geqq 2$,

(iii) $\left|\tilde{H}_{x}^{\prime}\right| \leqq L^{1+\alpha} \delta+O\left(e^{-\tilde{\beta}}\right)$,

$$
\left|\tilde{H}_{C^{\prime}}^{\prime}\right| \leqq e^{--2 \tilde{\beta}^{\prime}\left|C^{\prime}\right|} \quad \widetilde{\beta}^{\prime}=L^{1-\alpha} \widetilde{\beta},
$$

where (4.23) follows from

$$
|C|>\frac{L}{O(1)}\left|\left[L^{-1} C\right]\right| \quad \text { if } \quad d(C)>L / 4
$$

by taking $\alpha>0$ and $L$ large. Equation (4.22) shows that, upon blocking, $\tilde{H}^{\prime}$ may increase. Iterating the RGT, it would eventually be large enough to prevent us from doing the coarse graining. Thus we will introduce a new large field region $D^{\prime}$, adding to the previous one regions where $\tilde{H}^{\prime}$ is large. Let

$$
n_{x}^{\prime}=\chi\left(\left|\tilde{H}_{x}^{\prime}\right|>\delta^{\prime}\right)
$$

(we will let $\delta$ decrease during the iteration, see Sects. 5 and 7 , so that $\delta^{\prime}<\delta$ ) and put

$$
D^{\prime}=\left[L^{-1} D\right] \cup R
$$

with $R=\left\{x \mid n_{x}^{\prime} \neq 0\right\}$, and $D$ is defined by (3.6). We may now block the contours. Let

$$
\tilde{\Gamma}^{\prime}=\left[L^{-1} \underline{\Gamma}\right] \cup R
$$

and decompose $\tilde{\Gamma}^{\prime}=\cup \underline{\gamma}^{\prime}$ into connected components $\tilde{\gamma}^{\prime}$. (We put a tilde on $\tilde{\Gamma}, \tilde{\gamma}$ because they are not yet our new contours.) How do we block the signs $\sigma(\Gamma)$ ? We define $\sigma\left(\tilde{\gamma}^{\prime}\right)$ by the Majority rule: We just put $\sigma_{x}\left(\tilde{\gamma}^{\prime}\right)=1(-1)$ if $\sum_{y \in L x} \sigma_{y}(\Gamma)>0(<0)$ (remember that $L$ is odd), and $\tilde{\gamma}^{\prime} \equiv\left(\tilde{\gamma}^{\prime}, \sigma\left(\tilde{\gamma}^{\prime}\right)\right)$. We denote also $\tilde{\Gamma}^{\prime}=\left(\tilde{\gamma}^{\prime}\right)$. With this definition, $\tilde{\Gamma}^{\prime}$ is again compatible, since $\sigma\left(\tilde{\gamma}^{\prime}\right)$ is constant on connected components of $\tilde{\gamma}^{\prime c}$. However, $\sigma\left(\tilde{\gamma}^{\prime}\right)$ are rather arbitrary on $\underline{\tilde{\gamma}}$; unlike Ising contours, $\sigma$ need not 
change sign any more! Let us now proceed with the blocking of (4.14). We define the small field $H^{\prime}$ by

$$
H_{x}^{\prime}=f^{\prime}\left(\tilde{H}_{x}^{\prime}\right), \quad H_{C}^{\prime}=\tilde{H}_{C}^{\prime}, \quad|C|>1
$$

with $f^{\prime}(z)=z \chi\left(|z| \leqq \delta^{\prime}\right)$, and write

$$
Z^{\prime}=\sum_{\tilde{\Gamma}^{\prime} \supset D^{\prime}} e^{\beta^{\prime}\left(H^{\prime}, V^{-}\left(\tilde{\Gamma}^{\prime}\right)\right)} \sum_{\Gamma \supset D}^{\tilde{\Gamma}^{\prime}, l} \rho(\Gamma) e^{\beta S(\Gamma)}
$$

with, collecting terms,

$$
S(\Gamma)=-\left(\chi_{\Gamma} \delta h^{+}, V\right)-\left(\chi_{\Gamma} \delta H, V^{-}(\Gamma)\right)+\left(H+\delta H, V^{-}(\Gamma)\right)-L^{2-\alpha}\left(H^{\prime}, V^{-}\left(\tilde{\Gamma}^{\prime}\right)\right) .
$$

The $\sum^{\Gamma^{\prime}, l}$ just means that we sum large contours with the constraint $\left[L^{-1} \Gamma\right] \cup$ $R=\widetilde{\Gamma}^{\prime}$. The reader should realize that, if we did not have $S(\Gamma)$ in (4.27), we would already have the representation (4.1) for $Z$, with $\rho^{\prime}\left(\tilde{\gamma}^{\prime}\right)$ given by $\sum_{\Gamma}^{\hat{\gamma}, l} \rho(\Gamma)$. The rest of this section consists in finding corrections to this formula, due to $S(\Gamma)$.

Consider (4.28): it is a linear combination of $\delta h_{C}^{ \pm}$and $H_{x}$. The first two terms involve $\delta h_{C}^{ \pm}$such that $C \cap \underline{\Gamma} \neq \emptyset$ i.e.,

$$
C \cap L \tilde{\Gamma}^{\prime} \neq \emptyset \text {. }
$$

As for the last two terms, they cancel outside $L \tilde{\Gamma}^{\prime}$ except for some $\delta H_{C}$ in the definition of $\tilde{H}^{\prime}\left(=H^{\prime}\right.$ in $\left.\left(L \tilde{\Gamma}^{\prime}\right)^{c}\right)$ for which $C$ satisfies (4.29). Hence $S(\Gamma)$ contains $\delta h_{C}^{ \pm}$obeying (4.29) and $\bar{H}_{x}$ with $x \in L \tilde{\Gamma}^{\prime}$. So it represents interactions and self-interactions of the $\tilde{\gamma}^{\prime}$ s. We write

$$
S(\Gamma)=\sum_{C} U_{C}(\Gamma)+\sum_{\tilde{\gamma}^{\prime} \in \tilde{\Gamma}^{\prime}} E\left(\tilde{\gamma}^{\prime} \mid \Gamma\right) .
$$

The self-interaction $E(\tilde{\gamma} \mid \Gamma)$ has all terms with $C \cap L \tilde{\gamma}^{\prime} \neq \emptyset$ and $d(C)<L / 4$. Note that such $C$ 's, will not join two $L \tilde{\gamma}^{\prime}$ since they are at least a distance $L$ apart. Of course, $E\left(\tilde{\gamma}^{\prime} \mid \Gamma\right)$ depends only on the part $\tilde{\Gamma}$ of $\Gamma$ with $\tilde{\Gamma} \subset \tilde{\gamma}^{\prime} . U_{C}(\Gamma)$ collects the $\delta h_{\bar{C}}^{ \pm}$with $d(C)>L / 4$ [and, of course, satisfying (4.29)]. The form of this linear combination of $\delta h_{\bar{C}}^{ \pm}$depends only on the part of $\Gamma$ contained within $L \widetilde{\Gamma}_{C}^{\prime} \cap C$, where

$$
\tilde{\Gamma}_{C}^{\prime}=\left\{\tilde{\gamma}^{\prime} \in \tilde{\Gamma}^{\prime} \mid C \cap L \underline{\gamma}^{\prime} \neq \emptyset\right\} .
$$

Since $\delta h_{C}^{ \pm}$and $H_{C}^{\prime}$ are random variables defined for all $C \subset V$, we see from (4.28) that $U_{C}(\Gamma)$ is a random variable defined for arbitrary $C$ (being zero if the above constraints do not hold) and any $\Gamma, \tilde{\Gamma}^{\prime}$ with $\left[L^{-1} \Gamma\right] \subset \widetilde{\Gamma}^{\prime}$ (we use this notation here and below to mean $\left[L^{-1} \underline{\Gamma}\right] \subset \tilde{\Gamma}^{\prime}$ and $\sigma\left(\tilde{\Gamma}^{\prime}\right)=\sigma\left(\left[L^{-1} \Gamma\right]\right)$. Inserting (4.30) into (4.27) we have

$$
Z^{\prime}=\sum_{\tilde{\Gamma}^{\prime} \supset D^{\prime}} e^{\beta^{\prime}\left(H^{\prime}, V^{-}\left(\tilde{\Gamma}^{\prime}\right)\right)} \sum_{\Gamma \supset D}^{\tilde{\Gamma}^{\prime}, l} \rho(\Gamma) e^{\beta\left(\sum_{C} U_{C}(\Gamma)+\sum_{\tilde{\gamma}^{\prime}} E\left(\tilde{\gamma}^{\prime} \mid \Gamma\right)\right)} .
$$

The sum over $U_{C}$ is the only term in (4.32) not factorizing over $\tilde{\gamma}^{\prime} \in \tilde{\Gamma}^{\prime}$. We thus have to Mayer-expand it, in order to get the new activities $\rho^{\prime}$. It will be important later (in the proof of (6.2)) to preserve the positivity of $\rho^{\prime}$. $U_{C}$ can have an arbitrary sign as can $\exp \left(\beta U_{C}\right)-1$. Note however that, by (4.8),

$$
\left|U_{C}(\Gamma)\right| \leqq e^{-\tilde{\beta}|C| / 3}
$$


(independently of $\Gamma$ ). So we may define

$$
\tilde{W}_{C}\left(\tilde{\Gamma}^{\prime}\right)=\min _{\Gamma} U_{C}(\Gamma)
$$

with the minimum taken over all $\Gamma$ such that $\left[L^{-1} \Gamma\right] \subset \tilde{\Gamma}^{\prime} . \tilde{W}$ is a well defined random variable satisfying (4.33), and depends on $\tilde{\Gamma}^{\prime}$ only through $\tilde{\Gamma}_{C}^{\prime}$, defined in (4.31). So we write

$$
U_{C}(\Gamma)=\tilde{W}_{C}\left(\tilde{\Gamma}^{\prime}\right)+I_{C}(\Gamma)
$$

whence

$$
0 \leqq I_{C}(\Gamma) \leqq 2 e^{-\tilde{\beta} \mid C / / 3}
$$

Inserting (4.35) into (4.32) we get

$$
Z^{\prime}=\sum_{\tilde{I}^{\prime} \supset D^{\prime}} e^{\beta^{\prime}\left(H^{\prime}, V^{-}\left(\tilde{\Gamma}^{\prime}\right)\right)+\beta^{\prime}\left(W\left(\tilde{\Gamma}^{\prime}\right), V^{\prime}\right)} \sum_{\Gamma^{\prime} \supset D}^{\tilde{\Gamma}^{\prime}, l} \rho(\Gamma) \exp \left[\beta \sum I_{C}(\Gamma)+\beta \sum E\left(\tilde{\gamma}^{\prime} \mid \Gamma\right)\right],
$$

where $W\left(\tilde{\Gamma}^{\prime}\right)$ is the blocked $\tilde{W}$ :

$$
W_{C^{\prime}}\left(\tilde{\Gamma}^{\prime}\right)=L^{-2+\alpha} \sum_{\left[L^{-1} C\right]=C^{\prime}} \tilde{W}_{C}\left(\tilde{\Gamma}^{\prime}\right) .
$$

Then, we Mayer expand $I$ :

$$
\exp \beta \sum I_{C}=\prod_{C}\left(e^{\beta I_{C}}-1+1\right)=\sum_{\mathscr{C}} \prod_{C \in \mathscr{C}}\left(\exp \left(\beta I_{C}\right)-1\right) \equiv \sum_{\mathscr{C}} \phi(\mathscr{C}),
$$

which has positive terms due to (4.36). Inserting into (4.37), we have

$$
\sum^{\tilde{\Gamma}^{\prime}, l}=\sum_{\Gamma, \mathscr{C}}^{\tilde{\Gamma}^{\prime}, l} \tilde{\rho}(\Gamma) \phi(\mathscr{C})
$$

with

$$
\tilde{\rho}(\Gamma)=\rho(\Gamma) \exp \left(\beta \sum_{\tilde{\gamma}^{\prime}} E\left(\tilde{\gamma}^{\prime} \mid \Gamma\right)\right) .
$$

The last step now is to block $\Gamma \cup \mathscr{C}$, decompose the result into connected components and associate new activities to the later. Let

$$
\left[L^{-1}(\underline{\Gamma} \cup \mathscr{C})\right] \cup R=\underline{\Gamma}^{\prime}=\cup \underline{\gamma^{\prime}} .
$$

Note, that $\tilde{\Gamma}^{\prime} \subset \Gamma^{\prime}\left[\right.$ see $\left.\left(4.25^{\prime}\right)\right]$. Since $W$ depends only on $\tilde{\Gamma}^{\prime}$ we will slightly generalize our notion of a contour. We take $\gamma^{\prime}=\left(\underline{\gamma}^{\prime}, \sigma\left(\tilde{\Gamma}^{\prime}\right)\right)$, where $\tilde{\Gamma}^{\prime} \subset \underline{\gamma}^{\prime}$, and rewrite (4.37) with (4.40) in terms of such $\gamma^{\prime}$ :

$$
Z^{\prime}=\sum_{\Gamma^{\prime} \supset D^{\prime}} \rho^{\prime}\left(\Gamma^{\prime}\right) e^{\beta^{\prime}\left(H^{\prime}, V^{-}\left(\tilde{\Gamma}^{\prime}\right)\right)+\beta^{\prime}\left(W\left(\tilde{\Gamma}^{\prime}\right), V^{\prime}\right)},
$$

with $\Gamma^{\prime}=\left(\underline{\Gamma}^{\prime}, \sigma\left(\tilde{\Gamma}^{\prime}\right)\right)$ and $\tilde{\Gamma}^{\prime}$ constrained by $\tilde{\Gamma}^{\prime} \supset D^{\prime}\left(V^{-}\left(\tilde{\Gamma}^{\prime}\right)=V^{-}\left(\Gamma^{\prime}\right)\right)$. The activities are

$$
\rho^{\prime}\left(\gamma^{\prime}\right)=\sum_{\Gamma, \mathscr{C}}^{\tilde{\Gamma}^{\prime}, l} \tilde{\rho}(\Gamma) \phi(\mathscr{C})
$$

with $\underline{\Gamma} \supset D \cap L \underline{\gamma}^{\prime},\left[L^{-1} \underline{\Gamma}\right] \cup\left(R \cap \underline{\gamma^{\prime}}\right)=\tilde{\Gamma}^{\prime}$ and $\left[L^{-1}(\underline{\Gamma} \cup \mathscr{C})\right] \cup\left(R \cap \underline{\gamma}^{\prime}\right)=\underline{\gamma}^{\prime} ; \rho^{\prime}\left(\gamma^{\prime}\right)=0$ if the sum is empty. Equation (4.43) is the result of the first RGT. In the next section we show that this form is in fact stable under the RGT. 


\section{Flow of the RGT}

5.1 The Result. Let us assume that after $k$ steps of iteration of the RGT we have a partition function of the form (4.43), which was produced by the first step (we drop $k$ below and denote $k+1$ by prime):

$$
Z=\sum_{\Gamma} \rho(\Gamma) \exp \left[\beta\left(H, V^{-}(\Gamma)\right)+\beta W(\Gamma)\right] .
$$

$\Gamma$ is a compatible set of contours determined by $\underline{\Gamma}$ and another set $\tilde{\Gamma}$ such that $\Gamma=(\underline{\Gamma}, \sigma(\tilde{\Gamma}))$. Equation $(5.1)$ is constrained by $\widetilde{\Gamma} \supset D$. The variables are as follows:

$\rho(\gamma)$ are random variables with

(i) $\rho(\gamma)$ is independent of $h_{x}$, if $d\left(\underline{\gamma},\left[L^{-k} x\right]\right) \geqq 2$,

(ii) $\rho(\gamma)(h)=\rho(-\gamma)(-h)$ with $-\gamma \equiv(\underline{\gamma},-\sigma(\gamma))$,

(iii) $0 \leqq \rho(\gamma) \leqq \exp \left[-\tilde{\beta}|\underline{\gamma} \backslash \bar{D}|-\beta\left|\partial V^{-}(\gamma)\right|+\beta(N, \underline{\gamma})\right]$,

where $\bar{D}=\{x \mid d(x, D) \leqq 1\}$. The reason why we have $\bar{D}$ in (5.4) and not simply $D$ will become clear in Sect. 6 when we iterate (5.4).

[For $k=1$ (i) can readily be seen and for (ii), use (4.7) to show that $S(\Gamma)(h)=$ $S(-\Gamma)(-h)$. E.g., $\left(\chi_{\Gamma} \delta H, V^{-}(\Gamma)\right)=\left(\chi_{-r} \delta H, V^{+}(-\Gamma)\right)=\left(\chi_{-\Gamma} \delta H, V \backslash V^{-}(-\Gamma)\right)=$ $\left(\chi_{-\Gamma} \delta H(-h), V^{-}(-\Gamma)\right)-\left(\chi_{-\Gamma} \delta H(-h), V\right)$. The last term combines with the first in (4.28)]. $\beta$ and $\widetilde{\beta}$ will be given by

$$
\beta_{k}=L^{(2-\alpha) k} \beta, \quad \widetilde{\beta}_{k}=L^{(1-\alpha) k} \widetilde{\beta},
$$

where $\alpha$ can be chosen as small as one wishes, provided $L$ is large enough. (The only constraint being that $L^{\alpha}$ is larger than some geometrical constants.)

$H=\left\{H_{C}\right\}$ satisfies

$$
\begin{aligned}
& \text { (i) As in (5.2), with } \underline{\gamma} \text { replaced by } C \text {, } \\
& \text { (ii) } H_{C}(-h)=-H_{C}(h), \\
& \text { (iii) }\left|H_{C}\right| \leqq e^{-\tilde{\beta}|C|},\left|H_{x}\right| \leqq \delta_{k} \equiv(k+1)^{-2} \delta .
\end{aligned}
$$

Moreover, $H_{x}$ and $H_{y}$ are independent for $|x-y| \geqq 2$. Finally,

$$
W(\Gamma)=\sum W_{C}(\Gamma)
$$

with $W_{C}(\Gamma)=0$ unless $C \cap \tilde{I} \neq \emptyset$ and $C$ is connected. $W_{C}(\Gamma)$ depends on $h$ as in (5.2) (with $\underline{\gamma}$ replaced by $C$ ) and on $\Gamma$ only through $\tilde{\Gamma}_{\mathrm{C}}$. We have $W_{\mathrm{C}}(\Gamma)(h)=$ $W_{C}(-\Gamma)(-h)$ and

$$
\left|W_{C}(\Gamma)\right| \leqq e^{-\tilde{\beta}|C|}
$$

The purpose of this section is to reproduce (5.1) algebraically for $k+1$. In Sect. 6 we iterate the bound (5.4). The steps of the RGT are almost the same as in Sect. 4. The only novelty is that we want to forget about some of the region $D$, as explained in Sect. 3. This is done by means of the variables $N$ in (5.4) which keep track of the large fields created in previous $R$-regions and/or brought together by blocking. 
Thus, as before (see (3.6)), given $\left\{N_{x}\right\}_{x \in V}, D$ is defined by

$$
D=\left\{x \mid N_{x} \neq 0\right\} .
$$

We decompose $D$ into $L^{1 / 2}$ connected components, i.e., they correspond to the connected components of the graph on $D$ obtained by joining points which are at a distance $\leqq L^{1 / 2}$. A $L^{1 / 2}$ connected component $D_{i}$, of $D$, is small if

$$
\begin{aligned}
\left|D_{i}\right| & \leqq L^{(1-\alpha) / 2}, \\
d\left(D_{i}\right) & \leqq L / 4,
\end{aligned}
$$

and

$$
\sum_{y \in D_{1}} N_{y}<L L^{-k} \varepsilon^{2} .
$$

A connected component is large otherwise. The region of $D$ that we absorb in the small field is $\mathscr{D}=\bigcup_{i} D_{i}$, where the union is over all small components. The reasons for this rather complicated definition of $\mathscr{D}$ will become clear later.

Since $D_{i}$ are $L^{1 / 2}$ connected components, we have trivially

$$
d(\mathscr{D}, D \backslash \mathscr{D}) \geqq L^{1 / 2} .
$$

One fact about $\mathscr{D}$ that we shall use is: Let $C$ be a connected set with $d(C) \geqq L^{1 / 2}$, then

$$
|C \backslash \mathscr{D}| \geqq|C| / 2
$$

(for $L$ large enough). Equation (5.13) is obvious, because $C$ is connected in the usual sense, the components $D_{i}$ are at a distance $L^{1 / 2}$ from one another and each of them contains at most $L^{-\alpha / 2} L^{1 / 2}$ points. Another fact where we use the definition of $\mathscr{D}$ appears in Lemma 1 of Sect. 7.

Now we repeat the coarse graining and blocking operations of Sect. 4.

\subsection{Coarse Graining. Call $\gamma$ small if}

$$
d(\underline{\gamma}) \leqq L \quad \text { and } \quad V(\gamma) \cap(D \backslash \mathscr{D})=\emptyset,
$$

and large otherwise. Decompose $\Gamma$ into small and large contours and sum the former

$$
Z=\sum_{\tilde{I} \supset D \backslash \mathscr{D}}^{l} \rho(\Gamma) e^{\beta(H, V-(\Gamma))+\beta W(\Gamma)} Z_{s}(V \backslash \underline{\Gamma} \mid \Gamma),
$$

where

$$
Z_{s}(V \backslash \underline{\Gamma} \mid \Gamma)=\sum_{\Gamma_{s}}^{s} \rho\left(\Gamma_{s}\right) \exp \beta\left[\left(H, V^{-}\left(\Gamma_{s}^{+}\right)\right)-\left(H, V^{+}\left(\Gamma_{s}^{-}\right)\right)+W\left(\Gamma, \Gamma_{s}\right)\right],
$$

with $\Gamma_{s}$ summed over $V \backslash \underline{\Gamma} \supset \underline{\Gamma}_{s} \supset(\mathscr{D} \backslash \underline{\Gamma})$, and we split $\Gamma_{s}=\Gamma_{s}^{+} \cup \Gamma_{s}^{-}$with $\Gamma_{s}^{ \pm} \subset V^{ \pm}(\Gamma)$. Also, we wrote

$$
W\left(\Gamma \cup \Gamma_{s}\right)=W(\Gamma)+W\left(\Gamma, \Gamma_{s}\right) .
$$

Thus $W\left(\Gamma, \Gamma_{s}\right)=\sum_{C} W_{C}\left(\Gamma \cup \Gamma_{s}\right)-W_{C}(\Gamma)$ with $C \cap \Gamma_{s} \neq \emptyset$.

Next we exponentiate (5.15). This has two aspects. The $H$-part is bounded by $\beta\left|\partial V^{ \pm}\left(\Gamma_{s}\right)\right|$ and this is dominated by the $\beta\left|\partial V^{-}(\gamma)\right|$ terms in (5.4) since the $\gamma \in \Gamma_{s}$ are small, as defined above. 
The second aspect is that (5.15) has no $\underline{\Gamma}_{s} \neq \emptyset$ term if $\mathscr{D} \backslash \underline{\Gamma}$ is not empty. So we need to extract from $Z_{s}$ the $\rho(\gamma)$ 's that have no damping, i.e., those in $\overline{\mathscr{D}}$ (see (5.4)) with $\sigma(\gamma)$ constant. Thus write $\overline{\mathscr{D}}=\cup \overline{\mathscr{D}}_{i}$ in terms of ordinary connected components and define for any set $\mathscr{B}_{i}$ such that $\overline{\mathscr{D}}_{i} \supset \mathscr{B}_{i} \supset \mathscr{D} \cap \overline{\mathscr{D}}_{i}=\mathscr{D}_{i}$,

$$
\mathscr{Z}^{ \pm}\left(\mathscr{B}_{i}\right)=\sum_{\Gamma} \rho(\Gamma)
$$

with $\gamma \in \Gamma$ having $\underline{\gamma} \subset \mathscr{B}_{i}$ and $\sigma(\gamma)$ constant $( \pm)$. Now we write

$$
Z_{s}(V \backslash \underline{\Gamma} \mid \Gamma)=\prod^{\mathscr{Z}^{+}}\left(\mathscr{B}_{i}\right) \prod \mathscr{Z}^{-}\left(\mathscr{B}_{i}\right) \hat{Z}_{s}(V \backslash \underline{\Gamma} \mid \Gamma),
$$

where $\mathscr{B}_{i}=(V \backslash \bar{\Gamma}) \cap \overline{\mathscr{D}}_{i}$ and the products run over $\overline{\mathscr{D}}_{i} \subset V^{ \pm}(\Gamma) \backslash \Gamma$. (Note that such $\overline{\mathscr{D}}_{i}$ may touch $\underline{\Gamma}$ unlike $\mathscr{B}_{i}$; the point being, that the contours in $\hat{Z}_{s}$ cannot touch $\underline{\Gamma}$.)

$\hat{Z}_{s}$ may be Mayer expanded as in Sect. 4, the only difference being the presence of $W\left(\Gamma, \Gamma_{s}\right)$ which can be expanded, due to (5.8), and the division by the $\mathscr{Z}^{ \pm}$'s. For the latter, we use the lower bound in (6.2). The result is

$$
\hat{Z}_{s}(V \backslash \underline{\Gamma} \mid \Gamma)=\exp \beta\left[\left(\delta \tilde{h}^{+}, V^{+} \backslash \underline{\Gamma}\right)+\left(\delta \tilde{h}^{-}, V^{-} \backslash \underline{\Gamma}\right)+\delta W(\Gamma)\right]
$$

with the bounds

$$
\left|\delta \tilde{h}_{C}^{ \pm}\right|,\left|\delta W_{C}\right| \leqq\left\{\begin{array}{ll}
\exp (-\tilde{\beta} / 2) & d(C)<L / 4 \\
\exp (-\tilde{\beta}|C| / 4) & d(C) \geqq L / 4
\end{array} .\right.
$$

Equations (5.19) are obvious if we show that the expansion converges [which, itself follows easily from (5.13)]. See Sect. 6.3 for more details. Combine next $\delta \tilde{h}^{ \pm}$with $\mathscr{Z}^{ \pm}\left(\overline{\mathscr{D}}_{i}\right):$

$$
\delta h_{\bar{C}}^{ \pm}=\delta \tilde{h}_{C}^{ \pm}+1 / \beta \log \mathscr{Z}^{ \pm}\left(\overline{\mathscr{D}}_{i}\right) \delta_{C, \overline{\mathscr{D}}_{i}} .
$$

This satisfies, due to (5.12), the fact that $\mathscr{D}_{i} \subset \mathscr{D}$ and the bounds (6.1) and (6.2),

$$
\left|\delta h_{\bar{C}}^{ \pm}\right| \leqq c \varepsilon^{2} L^{-k+1}, \quad d(C)<L / 4
$$

(since $\exp (-\tilde{\beta} / 2) \ll \varepsilon^{2} L^{-k+1}$ ), and (5.19) for $d(C)>L / 4$. Insert (5.17'), (5.18) and (5.20) into (5.14) and pull out the + state contribution as in Sect. 4 to get

$$
\begin{aligned}
Z= & \exp \beta\left(\delta h^{+}, V\right) Z^{\prime}, \\
Z^{\prime}= & \sum_{\Gamma}^{l} \hat{\rho}(\Gamma) \exp \beta\left(H+\delta H, V^{-}\right) \\
& +\beta(W(\Gamma)+\delta W(\Gamma)) \exp \left[-\beta\left(\chi_{\Gamma} \delta h^{+}, V\right)-\beta\left(\chi_{\Gamma} \delta H, V^{-}\right)\right],
\end{aligned}
$$

where the sum is over sets of large contours with $\tilde{\tilde{I}} \supset D \backslash \mathscr{D}$, and

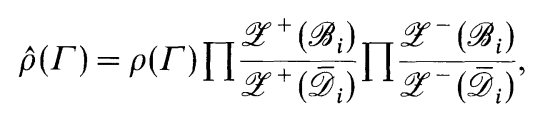

where the products run over $\mathscr{D}_{i} \subset V^{ \pm}(\Gamma) \backslash \underline{\Gamma}$. Note that these latter ratios are 1 unless $\overline{\mathscr{D}}_{i}$ is connected to $\underline{\Gamma}$.

5.3 Blocking. This will be almost identical to Sect. $4 . H^{\prime}$ is defined by (4.16), (4.17) with $H+\delta H$ replacing $\delta H$ in (4.17) and (4.26). Properties i), ii) (see (5.6)) and the first inequality in (5.7) hold with primes. The independence (4.21) is still true and, 
due to $(5.21),(4.22)$ is replaced by

$$
\left|\tilde{H}_{x}^{\prime}\right|<L^{1+\alpha} \delta+O\left(\varepsilon^{2} L^{-k}\right)
$$

$n^{\prime}$ is given by (4.24). Next, we block

$$
\tilde{\Gamma}^{\prime}=\left[L^{-1} \Gamma\right] \cup R
$$

with the majority rule included in [ ]. So, as in (4.27),

$$
Z^{\prime}=\sum_{\tilde{\Gamma}^{\prime} \supset D^{\prime}} \exp \left(\beta^{\prime}\left(H^{\prime}, V^{-}\left(\tilde{\Gamma}^{\prime}\right)\right)\right) \sum_{\Gamma}^{\tilde{\Gamma}^{\prime}, l} \hat{\rho}(\Gamma) e^{\beta S(\Gamma)}
$$

where now $\Gamma=(\underline{\Gamma}, \sigma(\tilde{\Gamma}))$ satisfies the constraints (5.24) and $\tilde{\Gamma} \supset D \backslash \mathscr{D}$. Notice that, because of the additional factors in $\left(5.22^{\prime}\right), \hat{\rho}(\Gamma)$ does not necessarily factorize over $\gamma \in \Gamma$ (different $\gamma$ 's may be connected to the same $\overline{\mathscr{D}}_{i}$ ). However, $\hat{\rho}(\Gamma)$ certainly factorizes over connected components of $\tilde{\Gamma}^{\prime}$. Indeed, the latter are at a distance at least $L$ from each other (before blocking) and it is clear that contours cannot be connected by $\overline{\mathscr{D}}$ over such a distance. $S(\Gamma)$ equals

$$
\begin{aligned}
S(\Gamma)= & W(\Gamma)+\delta W(\Gamma)-\left(\chi_{\Gamma} \delta h^{+}, V\right)-\left(\chi_{\Gamma} \delta H, V^{-}(\Gamma)\right) \\
& +\left(H+\delta H, V^{-}(\Gamma)\right)-L^{2-\alpha}\left(H^{\prime}, V^{-}\left(\widetilde{\Gamma}^{\prime}\right)\right) .
\end{aligned}
$$

It may be expanded as in (4.30), with $U_{C}(\Gamma)$ bounded now by

$$
\left|U_{C}(\Gamma)\right| \leqq \exp (-\tilde{\beta}|C| / 5)
$$

due to (5.19). Proceeding now exactly as in Sect. 4, we obtain (4.43), i.e., (5.1) with primes; (4.44), (4.41) and (4.39) define the new activities, with $\rho$ replaced by $\hat{\rho}$.

Let us finish with a note on $N^{\prime}$. We define

$$
N_{x}^{\prime}=L^{-2+\alpha} \sum_{y \in L x \backslash \mathscr{D}} N_{y}+n_{x}^{\prime} .
$$

Then, clearly, $D^{\prime}=\left\{x \mid N_{x}^{\prime} \neq 0\right\}$ (where $D^{\prime}=\left[L^{-1}(D \backslash \mathscr{D})\right] \cup R$, by definition).

\section{Inductive Bounds}

The goal of this section is to prove (5.4) for $\rho$, inductively in $k$ :

$$
0 \leqq \rho(\gamma) \leqq e^{-\tilde{\beta}|\underline{\gamma} \backslash \bar{D}|-\beta\left|\hat{\sigma} V^{-}(\gamma)\right|+\beta(N, \underline{\underline{\gamma}})}
$$

where $\bar{D}=\{x \mid d(x, D) \leqq 1)$, and a lower bound

$$
\mathscr{Z}^{ \pm}\left(\mathscr{B}_{i}\right) \geqq e^{-\beta\left(N, \overline{\mathscr{X}}_{i}\right)}
$$

for the partition functions (5.17). Let us recall here the formula for $\rho^{\prime}$ [see (4.44)]:

$$
\rho^{\prime}\left(\gamma^{\prime}\right)=\sum_{\Gamma, \mathscr{C}}^{\tilde{\Gamma}^{\prime}, l} \tilde{\rho}(\Gamma) \phi(\mathscr{C})
$$

with $\gamma^{\prime}=\left(\underline{\gamma^{\prime}}, \sigma\left(\tilde{\Gamma}^{\prime}\right)\right),\left[L^{-1} \Gamma\right] \cup R=\tilde{\Gamma}^{\prime},\left[L^{-1}(\underline{\Gamma} \cup \mathscr{C})\right] \cup R=\underline{\gamma}^{\prime}$ and $\underline{\Gamma} \supset D \backslash \mathscr{D}(R, D$, etc., are restricted to $\underline{\gamma}^{\prime}$ here and below). Here

$$
\tilde{\rho}(\Gamma)=\tilde{\rho}(\Gamma) \exp \left(\beta \sum_{\gamma^{\prime}} E\left(\tilde{\gamma}^{\prime} \mid \Gamma\right)\right)
$$


and

$$
\phi(\mathscr{C})=\prod_{C \in \mathscr{C}}\left(e^{\beta I_{C}(\Gamma)}-1\right) .
$$

6.1 The Upper Bound. The idea of the proof is easy; forget $\bar{D}$ for a moment. Then the scaling of $\beta, \widetilde{\beta}$ was explained in Sect. 3 and, for $\beta$, is justified by Lemma $1, \mathrm{~b}$. Note that the $\mathscr{C}$ part of $(6.3)$ carries $e^{-\tilde{\beta}}$. The iteration of $N,(5.28)$ absorbs the previous $N$ 's as well as $n_{x}^{\prime}$, in the $R$-region. Controlling the "entropy," i.e., the sum in (6.3) is easy, because each cube in $L \gamma^{\prime}$ gives at most a factor $\exp c L^{3}$ which can be absorbed, in $\beta, \tilde{\beta}$. Coming to $\bar{D}$, we have to absorb the part of $(N, \gamma)$ which is in $\mathscr{D}$. But that is controlled by $|\gamma \backslash \bar{D}|$ since, for $x$ in $\mathscr{D}, \beta N_{x} \ll \widetilde{\beta}$ and $\gamma$ is large. The reason why we iterate (6.1) with $\bar{D}$ instead of $D$ is explained in the proof of (6.16) below.

Equation (6.5) is bounded, using (5.27) and (4.35), (4.36), by

$$
0<\phi(\mathscr{C})<\exp [-\widetilde{\beta}|\mathscr{C}| / 6] \text {. }
$$

We recall that $E\left(\tilde{\gamma}^{\prime} \mid \Gamma\right)$ collects the $W_{C}(\Gamma), \delta W_{C}(\Gamma), \delta h_{C}^{ \pm}$and $H_{x}$ in $S(\Gamma)$ of $(5.26)$ satisfying [see the discussion after (4.30)]

$$
C \cap L \underline{\tilde{\gamma}}^{\prime} \neq \emptyset, \quad d(C)<\frac{L}{4} \quad \text { or } \quad x \in L \underline{\tilde{\gamma}}^{\prime} .
$$

By (5.8), (5.19) and (5.21), the contributions of the first four terms in (5.26) are bounded by

$$
c(L) \varepsilon^{2} L^{-k}|\underline{\Gamma} \cap \mathscr{D}|+\exp (-O(\widetilde{\beta}))\left|\underline{\gamma^{\prime}}\right|,
$$

where here and below $c(L)$ is a constant depending only on $L$. To control the last two terms, we use the fundamental result of our Majority rule.

Lemma 1. Let $\left[L^{-1} \Gamma\right]=\Gamma^{\prime}$. Then,

$$
\begin{aligned}
& \text { (a) }\left|V^{ \pm}\left(L \Gamma^{\prime}\right) \backslash V^{ \pm}(\Gamma)\right| \leqq c(L)\left|\partial V^{-}(\Gamma)\right|, \\
& \text { (b) }\left|\partial V^{-}(\Gamma)\right| \geqq c L^{2}\left|\partial V^{-}\left(\Gamma^{\prime}\right)\right| .
\end{aligned}
$$

(a) says the intuitively obvious fact that the blocked signs may differ from the unblocked ones only proportionally to the region where the latter are not constant and (b), that such regions are essentially two-dimensional. For a proof of (b), see [16]. So, $\left(H+\delta H, V^{-}(\Gamma)\right)$ differs from $L^{-2+\alpha}\left(H^{\prime}, V^{-}\left(\tilde{\Gamma}^{\prime}\right)\right)$ on a region proportional to $L R \cup \partial V^{-}(\Gamma)$, and thus, altogether, $\rho^{\prime}$ of $(6.3)$ is bounded by

$$
\begin{aligned}
\rho^{\prime}\left(\gamma^{\prime}\right) \leqq & \sum_{\Gamma, \mathscr{C}}^{\tilde{\Gamma}^{\prime}, l} \exp c(L) \beta\left[\delta|R|+\delta\left|\partial V^{-}(\Gamma)\right|+\varepsilon^{2} L^{-k}|\mathscr{D}|+e^{-O(\tilde{\beta})}\left|\underline{\gamma^{\prime}}\right|\right] \\
& \cdot \exp \left[-\frac{\tilde{\beta}}{6}|\mathscr{C}|-\tilde{\beta}|\underline{\Gamma} \backslash \bar{D}|-\beta \mid \partial V^{-}(\Gamma)+\beta(N, \underline{\Gamma})\right],
\end{aligned}
$$

where the last exponential comes from (6.6) and the induction and we write $\mathscr{D}$ for simplicity to denote the components of $\overline{\mathscr{D}}$ connected to $\underline{\Gamma}$. Moreover, the $|\mathscr{D}|$-term in (6.10) also controls the upper and lower bound on $\mathscr{Z}^{ \pm}\left(\mathscr{B}_{i}\right), \mathscr{Z}^{ \pm}\left(\overline{\mathscr{D}}_{i}\right)$ occurring in $\hat{\rho}$ (see $\left.\left(5.22^{\prime}\right)\right)$. For the upper bound, we have, from $(6.1) \exp (\beta(N, \mathscr{D}))$ for each term in (5.17) and $\exp (c(L)|\mathscr{D}|)$ for the number of terms in the sum (5.17). From 
(6.2) we have an upper bound $\exp (\beta(N, \mathscr{D}))$ on $\left(\mathscr{Z}^{ \pm}\right)^{-1}$. Now, $(N, \mathscr{D}) \leqq c L^{-k} \varepsilon^{2}|\mathscr{D}|$ follows from (5.12). Moreover, $c(L)|\mathscr{D}| \ll \beta_{k} L^{-k} \varepsilon^{2}|\mathscr{D}|$ because of the definition of $\beta_{k}$, (5.5). We proceed in steps to bound the exponents in (6.10). First, for $c(L) \delta<1$, we get

$$
c(L) \delta|R| \leqq \sum_{x \in \underline{\gamma}^{\prime}} n_{x}^{\prime} .
$$

Then, since $\left[L^{-1} \Gamma\right] \cup R=\tilde{\Gamma}^{\prime},(6.9)$ gives $\left|\partial V^{-}(\Gamma)\right|>c L^{2}\left|\partial V^{-}\left(\tilde{\Gamma}^{\prime}\right)\right|$, and since $\sigma\left(\gamma^{\prime}\right)=\sigma\left(\tilde{\Gamma}^{\prime}\right)$ gives $\partial V^{-}\left(\tilde{\Gamma}^{\prime}\right)=\partial V^{-}\left(\gamma^{\prime}\right)$, we have

$$
\beta(1-c(L) \delta)\left|\partial V^{-}(\Gamma)\right| \geqq c L^{2} \beta(1-c(L) \delta)\left|\partial V^{-}\left(\gamma^{\prime}\right)\right| .
$$

So the exponent in (6.10) is bounded from above by

$$
\begin{aligned}
& -\hat{\beta}\left|\partial V^{-}\left(\gamma^{\prime}\right)\right|+\beta(N, \underline{\Gamma})+\beta\left(n^{\prime}, \underline{\gamma}^{\prime}\right)+c(L) \beta \varepsilon^{2} L^{-k}|\mathscr{D}| \\
& \quad+e^{-O(\tilde{\beta})}\left|\underline{\gamma^{\prime}}\right|-\widetilde{\beta}|\mathscr{C}| / 6-\tilde{\beta}|\underline{\Gamma} \backslash \bar{D}|,
\end{aligned}
$$

where

$$
\hat{\beta}=c L^{2} \beta(1-c(L) \delta)
$$

By (5.28) and (5.12),

$$
\beta(N, \underline{\Gamma})+\beta\left(n^{\prime}, \underline{\gamma}^{\prime}\right) \leqq L^{2-\alpha} \beta\left(N^{\prime}, \underline{\gamma}^{\prime}\right)+L \beta \varepsilon^{2} L^{-k}|\underline{\Gamma} \cap \mathscr{D}| .
$$

The last term can be absorbed into the $\mathscr{D}$ term in (6.13). The only slightly subtle steps in the proof deal with the structure of $\mathscr{D}$, entering into the bound,

$$
|\underline{\Gamma} \backslash \bar{D}| \geqq c(|\underline{\Gamma} \backslash(\overline{D \backslash \mathscr{D}})|+|\overline{\mathscr{D}} \cap \underline{\Gamma}|),
$$

and the bar in $\bar{D}$, entering in

$$
c(\tilde{\beta}|\mathscr{C}|+\tilde{\beta}|\underline{\Gamma} \backslash(\overline{D \backslash \mathscr{D}})|) \geqq c^{\prime} L \tilde{\beta}\left|\underline{\gamma^{\prime}} \backslash \overline{D^{\prime}}\right| .
$$

Assuming these for a moment, we may finish the proof. Namely, (6.13) is now bounded by

$$
-\tilde{\beta}\left|\partial V^{-}\left(\gamma^{\prime}\right)\right|+L^{2-\alpha} \beta\left(N^{\prime}, \underline{\gamma}^{\prime}\right)-c^{\prime} L \widetilde{\beta}\left|\underline{\gamma}^{\prime} \backslash \bar{D}^{\prime}\right|+e^{-O(\tilde{\beta})}\left|\underline{\gamma^{\prime}}\right|,
$$

since we may absorb the $|\mathscr{D}|$-term into (6.15). This works, because $\varepsilon^{2} \beta L^{-k} \ll \widetilde{\beta}$ (see (5.5)).

We also have to control the sum in (6.10). This is at most $\exp \left(c(L)\left|\gamma^{\prime}\right|\right)$, because $\underline{\Gamma}, \mathscr{C}$, are subsets of $L \underline{\gamma}^{\prime}$. The choices of the different subsets $\underline{\Gamma} \subset \underline{\Gamma}$ is obviously included in that upper bound.

So, finally, we have as upper bound on (6.10), (6.17) with $c(L)\left|\underline{\gamma^{\prime}}\right|$ replacing the last term. Now bound

$$
\left|\underline{\gamma^{\prime}}\right| \leqq\left|\underline{\gamma^{\prime}} \backslash \overline{D^{\prime}}\right|+c\left|D^{\prime}\right|
$$

The first term in (6.18) can be combined with the third one in (6.17), lowering $c^{\prime}$. For the second term in (6.18) we absorb it in the second one in (6.17) as follows: we shall show in Sect. 7 (see (7.21)) that,

$$
(N, D) \geqq c^{k} L^{-3 k / 2} \varepsilon^{2}|D|
$$

for $c=27$. (The same inequality holds for any ordinary connected component of 
D.) Now let

$$
\beta^{\prime}=\beta\left(L^{2-\alpha}+0\left(L^{-(1-2 \alpha) k / 2}\right)\right)
$$

Then,

$$
c(L)\left|D^{\prime}\right|+L^{2-\alpha} \beta\left(N^{\prime}, \underline{\gamma^{\prime}}\right) \leqq \beta^{\prime}\left(N^{\prime}, \underline{\gamma}^{\prime}\right) .
$$

Inserting (6.18) and the last inequality into (6.17), we have [see $\left.\left(6.13^{\prime}\right)\right] \hat{\beta}>\beta^{\prime}$ and (6.17) is bounded from above by

$$
-\beta^{\prime}\left|\partial V^{-}\left(\gamma^{\prime}\right)\right|+\beta^{\prime}\left(N^{\prime}, \underline{\gamma}^{\prime}\right)-\tilde{\beta}^{\prime}\left|\underline{\gamma^{\prime}} \backslash \bar{D}^{\prime}\right|,
$$

where we use $L^{-\alpha}$ in $\widetilde{\beta}^{\prime}=L^{1-\alpha} \widetilde{\beta}$ to absorb the constant in (6.17). The redefinition, $\left(6.19^{\prime}\right)$, of $\beta^{\prime}$ [compare with (5.5)] is harmless since

$$
\prod_{k=1}^{\infty}\left(1+O\left(L^{-(1-2 \alpha) k / 2}\right)=O(1)\right.
$$

and does not affect any of the arguments where we use the approximation (5.5).

Combining (6.10) and (6.20), (6.1) with primes follows.

We still have to prove (6.15) and (6.16). For (6.15), it is enough to show that, for any $\gamma \in \Gamma$,

$$
|\underline{\gamma} \backslash \bar{D}| \geqq c|\underline{\gamma} \cap \overline{\mathscr{D}}| \text {. }
$$

Assume first that $\underline{\gamma} \cap \overline{(D \backslash \mathscr{D})}=\emptyset$, in which case (6.21) reduces to $|\underline{\gamma} \backslash \overline{\mathscr{D}}| \geqq c|\underline{\gamma} \cap \overline{\mathscr{D}}|$ (recall that, by $\left(5.12^{\prime}\right), \overline{D \backslash \mathscr{D}}=\bar{D} \backslash \overline{\mathscr{D}}$ ). Now, remember that $\gamma$ is large. If $d(\underline{\gamma})>L$, (6.21) follows from (5.13), since $\gamma$ is connected. Now, if $V(\gamma) \cap(D \backslash \mathscr{D}) \neq \bar{\emptyset}$, but $\underline{\gamma} \cap \overline{\mathscr{D}} \neq \emptyset$ (otherwise (6.21) is trivial) then, since $\underline{\gamma}$ is connected, $d(\underline{\gamma}) \geqq L^{1 / 2}$ because $\bar{d}(D \backslash \mathscr{D}, \mathscr{D}) \geqq L^{1 / 2}\left(5.12^{\prime}\right)$ and (6.21) follows again from (5.13).

Now, in general, $\underline{\gamma} \cap \overline{(D \backslash \mathscr{D})} \neq \emptyset$, so let $\underline{\gamma \backslash \overline{(D \backslash \mathscr{D})}}=\cup_{i} X_{i}$, where the $X_{i}$ 's are the connected components. Again because $\overline{D \backslash \mathscr{D}}$ and $\mathscr{D}$ are at least a distance $L^{1 / 2}$ apart, $d\left(X_{i}\right) \geqq c L^{1 / 2}$ if $X_{i} \cap \overline{\mathscr{D}} \neq \emptyset$, and we may apply (5.13).

Turning to the proof of (6.16), the reason for putting the bar in $\bar{D}$ is that, if we write $\underline{\Gamma} \backslash \overline{(D \backslash \mathscr{D})}=\bigcup_{i} X_{i}$, where $X_{i}$ are the connected components, then small $X_{i}$ 's [to which one could not apply $\left(4.23^{\prime}\right)$ ] are automatically in $L \bar{D}^{\prime}$, i.e., we claim that, if $d\left(X_{i}\right) \leqq L / 4$ then $X_{i} \subset L \bar{D}^{\prime}$. To see this, let $X_{i} \subset \gamma$ for some $\gamma \in \Gamma$. Since $\gamma$ is large we get that $V(\gamma) \cap(D \backslash \mathscr{D}) \neq \emptyset$ (otherwise, $\underline{\gamma} \cap(D \backslash \mathscr{D})=\emptyset$, but then $\underline{\gamma} \subset \bar{X}_{i}$ and $d\left(X_{i}\right) \leqq L / 4$ contradicts the fact that $\gamma$ is large). Now it is geometrically obvious that if $d\left(X_{i}\right) \leqq L / 4, X_{i} \subset \gamma, V(\gamma) \cap(D \backslash \mathscr{D}) \neq \emptyset$, then $X_{i} \subset L \bar{D}^{\prime}$ (using $L D^{\prime} \supset D \backslash \mathscr{D}$ ).

The rest is easy: the left-hand side of (6.16) is larger than

$$
c \widetilde{\beta}\left(\sum_{C \in \mathscr{C}}|C|+\sum_{d\left(X_{\imath}\right) \geqq L / 4}\left|X_{i}\right|\right),
$$

which, since $d(C) \geqq L / 4$ too $\left(I_{C}\right.$ in (4.35) collected precisely such $C$ 's), is larger than

$$
c^{\prime} \tilde{\beta} L \mid\left[L^{-1}\left(\mathscr{C} \cup\left(\bigcup_{d\left(X_{\imath}\right) \geqq L / 4} X_{i}\right)\right] \mid\right.
$$

by $\left(4.23^{\prime}\right)$. And, by the preceding discussion, this is bigger (since $R \subset D^{\prime}$ ) than

$$
c \tilde{\beta} L\left|\left[L^{-1}(\mathscr{C} \cup \underline{\Gamma})\right] \backslash \bar{D}^{\prime}\right| \geqq c \widetilde{\beta} L\left|\underline{\gamma^{\prime}} \backslash \bar{D}^{\prime}\right| .
$$


6.2 The Lower Bound. The lower bound (6.2) is much easier. By positivity of the $\rho$ 's, it is enough to prove that for an arbitrary component $D_{i}$ of $D$ :

$$
\rho\left(D_{i}^{ \pm}\right) \geqq e^{-\beta\left(N, D_{i}\right)},
$$

where $D_{i}^{ \pm}=\left(D_{i}, \pm 1\right)$. Let us show it inductively. In the first step, we have (see (3.10), (3.19))

$$
\rho\left(D_{i}^{ \pm}\right)=e^{ \pm \beta\left(h . D_{i}\right)}
$$

satisfying (6.22). Inductively, use (6.3) and note that all terms are positive. So consider the term $\mathscr{C}=\emptyset, \underline{\Gamma}=(D \backslash \mathscr{D}) \cap L D_{i}^{\prime}, \sigma(\Gamma)=$ constant. We get

$$
\rho^{\prime}\left(D_{i}^{\prime \pm}\right) \geqq \hat{\rho}(\Gamma) \exp \left(\beta E\left(D_{i}^{\prime \pm} \mid \Gamma\right)\right) .
$$

First we claim that, for the $\Gamma$ we have chosen, $\hat{\rho}(\Gamma)=\rho(\Gamma)$ (see $\left.\left(5.22^{\prime}\right)\right)$. Indeed, $\overline{\mathscr{D}}$ is not connected to $\overline{D \backslash \mathscr{D}}$ because of the distance between $\mathscr{D}$ and $D \backslash \mathscr{D}$ (see $\left.\left(5.12^{\prime}\right)\right)$. Next, we use (6.8) to bound

$$
E\left(D_{i}^{\prime \pm} \mid \Gamma\right) \geqq-e^{-O(\tilde{\beta})}\left|D_{i}^{\prime}\right|-c(L) \beta \delta\left|R \cap D_{i}^{\prime}\right|,
$$

where the second term comes from the last two terms in $(5.26)$ (here $\left.\partial V^{-}(\Gamma)=\emptyset\right)$, and the first term in (6.8) is absent because $\underline{\Gamma} \cap \mathscr{D}=\emptyset$.

Now we use the induction on $\rho(\Gamma),(6.11)$ and (6.14) (where the last term is again absent). And we use the redefinition of $\beta,(6.19)$, to absorb the $\left|D_{i}^{\prime}\right|$ term in (6.23). These two steps yield (6.22) with primes.

6.3 Contour Expansions. Let us now discuss the small contour expansion yielding (5.18) and (5.19) (the one used in Sect. 4 is a special case). We Mayer-expand the $W\left(\Gamma, \Gamma_{s}\right)$ in (5.15):

$$
e^{\beta W\left(\Gamma, \Gamma_{s}\right)}=\sum_{\mathscr{C}} \prod_{C \in \mathscr{C}}\left(e^{\beta\left(W_{C}\left(\Gamma \cup \Gamma_{s}\right)-W_{C}(\Gamma)\right)}-1\right) \equiv \sum_{\mathscr{C}} \psi(\mathscr{C}),
$$

where only $C \cap \underline{\Gamma}_{s} \neq \emptyset$ appear. Next, for a $\gamma \in \Gamma_{s}^{\sigma}(\sigma= \pm)$, let $\gamma^{\sigma} \equiv \underline{\gamma} \cup V^{-\sigma}(\gamma)$, divide the set

$$
\mathscr{C} \cup\left(\bigcup_{\gamma \in \Gamma_{s}} \gamma^{\sigma}\right)=\bigcup_{\alpha} Y_{\alpha}
$$

into connected components $Y_{\alpha}$ and write

$$
Z_{s}(V \backslash \Gamma \mid \Gamma)=\sum_{\left\{Y_{\alpha}\right\}} \prod A\left(Y_{\alpha}\right) .
$$

The constraint on $\left\{Y_{\alpha}\right\}$ is, that $\cup Y_{\alpha} \supset \mathscr{D} \backslash \underline{\Gamma}$, and $Y_{\alpha} \cap(\mathscr{D} \backslash \underline{\Gamma})$ is a (possibly empty) union of connected components of $\mathscr{D}$.

The activities $A\left(Y_{\alpha}\right)$ are

$$
A(Y)=\sum_{\Gamma_{s}, \mathscr{C}}^{s} \rho\left(\Gamma_{s}\right) e^{\beta\left[\left(H, V^{-}\left(\Gamma_{s}^{+}\right)\right)-\left(H, V^{+}\left(\Gamma_{s}^{-}\right)\right)\right]} \psi(\mathscr{C})
$$

with obvious constraints. To bound (6.27), note, that since both the contours and the fields are small, the $H$-part is bounded by $\beta\left|\partial V^{-}(\gamma)\right|$ in (5.4) $(\delta|V| \leqq|\partial V|$ if $d(V)<L$ and $\delta$ is small). Using (5.8) to bound $\psi(\mathscr{C})$ and the inequality $\beta\left|\partial V^{-\sigma}(\gamma)\right| \gg$ 
$\tilde{\beta}\left|V^{-\sigma}(\gamma)\right|$ (which holds because of the relation between $\beta$ and $\widetilde{\beta}$ ), we get

$$
|A(Y)| \leqq \exp \left[-a \widetilde{\beta}|Y \backslash \mathscr{B}|+c \varepsilon^{2} \beta L^{-k}|Y \cap \mathscr{B}|\right],
$$

where $\mathscr{B}=\cup \mathscr{B}_{i}$, and $a<\frac{1}{2}$. The last term in (6.28) comes from the $\mathscr{D}(\subset D)$ part of the upper bound, (6.1), on $\rho\left(\Gamma_{s}\right)$. Then we use (5.12).

Since $\varepsilon^{2} \beta_{k} L^{-k} \ll \widetilde{\beta}_{k}$ we can improve (6.28): if $Y \backslash \mathscr{B} \neq \emptyset$ the second term may be absorbed into the first one and, for $Y \subset \mathscr{B}_{i}$,

$$
A(Y)=\rho\left(Y^{\sigma}\right)+\mathcal{O}\left(e^{-a \tilde{\beta}}\right),
$$

where, in the first term, $\mathscr{C}=\emptyset$ and $Y^{\sigma}=(Y, \sigma)$ if $\mathscr{B}_{i} \subset V^{\sigma}(\Gamma)$.

Now we may discuss $\hat{Z}_{s}$ given by (5.17'). We decompose $\cup Y_{\alpha} \cup \mathscr{B}=\cup X_{\beta}$ into connected components and regroup

$$
Z_{s}(V \backslash \underline{\Gamma} \mid \Gamma)=\sum_{\left\{X_{\alpha}\right\}} \prod B\left(X_{\alpha}\right) .
$$

Now, for $X_{\alpha} \backslash \mathscr{B} \neq \emptyset$, we have (by lowering $a$ )

$$
\left|B\left(X_{\alpha}\right)\right| \leqq e^{-a \tilde{\beta}\left|X_{\alpha} \backslash \mathscr{B}\right|},
$$

and, using $\rho\left(Y^{\sigma}\right) \leqq \exp \left(\mathcal{O}\left(\varepsilon^{2}\right) \beta_{k} L^{-k}\right)$,

$$
\left|B\left(\mathscr{B}_{i}\right)-\mathscr{Z}^{\sigma}\left(\mathscr{B}_{i}\right)\right| \leqq \exp [-a \tilde{\beta}] .
$$

Using the lower bound (6.2) and (5.12) we see that $\mathscr{Z}^{ \pm}\left(\mathscr{B}_{i}\right)^{-1} \leqq \exp \mathcal{O}\left(\varepsilon^{2}\right) \beta L^{-k}$, and thus

$$
B\left(\mathscr{B}_{i}\right) \mathscr{Z}^{\sigma}\left(\mathscr{B}_{i}\right)^{-1}=1+\mathcal{O}\left(e^{-a \tilde{\beta}}\right)
$$

and $B\left(X_{\alpha}\right) \prod_{i} \mathscr{Z}^{ \pm}\left(\mathscr{B}_{i}\right)^{-1}$, with the product running over $\mathscr{B}_{i} \subset X_{\alpha}$, satisfies (6.31). The $X_{\alpha}$ in (6.30) are constrained to include $\mathscr{B}_{i}$ if intersected by it. Equation (6.33) shows that we may write for $\hat{Z}_{s}$ an expansion without this constraint and polymers bounded by (6.31) or by $e^{-a \vec{\beta}}$. In this new expansion the weight of $\mathscr{B}_{i}$ is simply $B\left(\mathscr{B}_{i}\right) \mathscr{Z}^{\sigma}\left(\mathscr{B}_{i}\right)^{-1}-1$.

The bound (5.19) for small $C$ is now immediate. For $d(C) \geqq L / 4$ we have damping only outside $\overline{\mathscr{D}}$. But using (5.13), the desired bound is obtained.

\section{Flow of the Effective Random Field}

7.1 The Result. In this section we establish properties of the effective random field - the $H$ and the $N$-needed in the discussion of the $h$-averages. Basically our analysis shows that the effective field behaves like a Gaussian of covariance $L^{-(1-\eta) k} \varepsilon^{2} \equiv \varepsilon_{k}^{2}$, although very small $N$ 's have modified behavior. ( $\eta$ may be chosen $=4 \alpha$, i.e., is as small as desired for $L$ large.)

Let us recall first the recursive definitions of $H$ and $N$. We had, for $H$

$$
\begin{aligned}
H_{x}^{k} & =\widetilde{H}_{x}^{k} \chi\left(\left|\tilde{H}_{x}^{k}\right|<\delta_{k}\right), \\
\tilde{H}_{x}^{k} & =L^{-2+\alpha} \sum_{y \in L x} H_{y}^{k-1}+q_{x}^{k},
\end{aligned}
$$

with the initial condition

$$
\tilde{H}_{x}^{0}=-2 h_{x},
$$


and $\delta_{k}=(k+1)^{-2} \delta . q^{k}$ is a shorthand for the non-linear contribution to (4.16) [defined by (4.13), (5.20)], and,

(i) $q^{k}$ is odd in $h$, i.e., $q^{k}(h)=-q^{k}(-h)$,

(ii) $\left|q_{x}^{k}\right| \leqq c(L) L^{-k} \varepsilon^{2}$,

(iii) $q_{x}^{k}, q_{y}^{k}$ are independent if $|x-y| \geqq 2$.

In particular (iii) implies, that

(iv) $H_{x}^{k}$ and $H_{y}^{k}$ are independent for $|x-y| \geqq 2$.

The solution of the recursion relation (7.1), (7.2) will be:

Proposition 1 The generating functional of $H^{k}$ satisfies

$$
\left\langle e^{t H_{\lambda}^{k}}\right\rangle \leqq e^{2 t^{2} \varepsilon_{k}^{2}} \quad \text { for } \quad|t| \leqq \delta \varepsilon_{k}^{-2} \equiv \delta L^{(1-\eta) k} \varepsilon^{-2},
$$

where $\langle>$, in this section, will denote the h-average.

Equation (7.4) will allow us to study the probability of $\tilde{H}_{x}^{k}$ given by (7.2) to be larger than $\delta_{k}$.

For $N$ we had [see (3.3), (3.5)]

$$
N_{x}^{0} \equiv n_{x}^{0}=\sum_{n=1}^{\infty}(n+1) \chi\left(\left|\tilde{H}_{x}^{0}\right| \in[n, n+1] \delta\right),
$$

and the recursion $[$ see $(5.28)]$

$$
N_{x}^{k}=L^{-2+\alpha} \sum_{y \in L x \backslash \mathscr{I}^{k-1}} N_{y}^{k-1}+n_{x}^{k}
$$

with

$$
n_{x}^{k}=\chi\left(\left|\tilde{H}_{x}^{k}\right|>\delta^{k}\right),
$$

$D^{k}=\left\{x \mid N_{x}^{k} \neq 0\right\}$ and $\mathscr{D}$ is defined after (5.10). Note, again, that

(v) $N_{x}^{k}$ and $N_{y}^{k}$ are independent for $|x-y| \geqq 2$.

The main result of our analysis is

Proposition 2. There is a constant $c$ such that for all $x$,

$$
P\left(N_{x}^{k} \neq 0 \text { for some } k\right) \leqq \exp \left(-\frac{c}{\varepsilon^{2}}\right) .
$$

Here $P$ is in the $h$-ensemble. More detailed knowledge of $N_{x}^{k}$ for fixed $k$ will be derived below.

7.2 The Small Fields. Why are we interested in the distribution of $H$, since it is small anyway? The reason is that we want to prove the unlikeliness of $n_{x}^{k}=1$, the basic input to $N$. Thus, assuming Proposition 1, let us first show that

$$
P\left(n_{x}^{k}=1\right) \leqq \exp \left[-\frac{\mathcal{O}(1) \delta_{k}^{2}}{\varepsilon_{k}^{2}}\right] .
$$

Equation (7.9) is of course a trivial consequence of the Gaussian bound (7.4) (we 
drop $k)$ :

$$
\begin{aligned}
P\left(n_{x}=1\right) & \leqq 2 P\left(\tilde{H}_{x}>\delta\right) \quad(\tilde{H} \text { is odd }) \\
& \leqq 2 e^{-t \delta}\left\langle e^{t \tilde{H}_{x}}\right\rangle \\
& \leqq 2 \exp \left[-t \delta+2 t^{2} \varepsilon^{2}\right] \\
& \leqq 2 \exp \left(-\delta^{2} / 8 \varepsilon^{2}\right) \quad\left(t=\delta / 4 \varepsilon^{2}\right)
\end{aligned}
$$

which implies (7.9) since $\delta^{2} / \varepsilon^{2}$ is large. To prove (7.4) we proceed inductively. For $k=0, \tilde{H}_{x}^{0}=-2 h_{x}$ and (7.4) is an equality. Thus assuming it for $k-1$, we use Schwartz inequality first to get (drop $k-1$ and replace $k$ by prime)

$$
\left\langle e^{t \tilde{H}_{x}^{\prime}}\right\rangle \equiv\left\langle\exp t\left[L^{-2+\alpha} \sum_{y \in L x} H_{y}+q_{x}\right]\right\rangle \leqq\left\langle\exp 2 t L^{-2+\alpha} \sum H_{y}\right\rangle^{1 / 2}\left\langle e^{2 t q}\right\rangle^{1 / 2} .
$$

For the first factor we use the independence (iv) of the $H^{\prime}$ 's. If the $L^{3} H_{y}$ 's were totally independent, we would of course get the bound $4 t^{2} L^{3} L^{-4+2 \alpha} \varepsilon_{k-1}^{2}$ allowing us to shrink $\varepsilon$.

However $H_{x}$ and $H_{y}$ are independent only for $|x-y| \geqq 2$. Thus divide $L x$ into $m$ subsets $U_{i}$ such that in $U_{i}$ the points have distance $\geqq 2$ between each other (in fact $m=27)$. By Schwartz, we get

$$
\begin{aligned}
\left\langle\sum_{y \in L x} \exp 2 t L^{-2+\alpha} H_{y}\right\rangle^{1 / 2} & \leqq \prod_{i=1}^{m}\left\langle\prod_{y \in U_{i}} \exp 2^{m+1} t L^{-2+\alpha} H_{y}\right\rangle^{2^{-m-1}} \\
& \leqq \exp 2^{-m-1} L^{3} \cdot 2\left(2^{m+1} t L^{-2+\alpha}\right)^{2} \varepsilon^{2} \leqq \exp t^{2} \varepsilon^{\prime 2} .
\end{aligned}
$$

The second inequality holds, by induction, as long as $2^{m+1} t L^{-2+\alpha} \leqq \delta \varepsilon^{-2}$, i.e., for $L$ large, it holds if $t \leqq \delta^{\prime}\left(\varepsilon^{\prime}\right)^{-2}$. Moreover, the last inequality holds if $L^{-1+2 \alpha} 2^{m+2} \leqq$ $L^{-1+\eta}$. Then take $\eta>2 \alpha$ and $L$ big. For the second factor in (7.10), note that (ii) implies $|t| q \ll 1$ for $|t| \leqq \delta L^{(1-\eta) k} \varepsilon^{-2}$, and therefore

$$
\left\langle e^{2 t q}\right\rangle \leqq 1+3 t^{2}\left\langle q^{2}\right\rangle,
$$

since $\langle q\rangle=0$ by (i). But $t^{2} q^{2} \ll t^{2} \varepsilon_{k}^{2}$, and thus (7.12) is a small correction to (7.11). We got $\left\langle e^{t \tilde{H}_{x}^{\prime}}\right\rangle \leqq e^{2 t^{2} \varepsilon^{\prime 2}}$. Now we may finish by: (write $\chi=\chi\left(\left|\tilde{H}^{\prime}\right|<\delta^{\prime}\right), \chi^{c}=1-\chi$ )

$$
\begin{aligned}
\left\langle e^{t H^{\prime}}\right\rangle & =\left\langle e^{t \tilde{H}^{\prime}} \chi+\chi^{c}\right\rangle \quad\left(H^{\prime}=0 \quad \text { on } \chi^{c}\right) \\
& =\left\langle e^{t \tilde{H}^{\prime}}\right\rangle+\left\langle\left(1-e^{t \tilde{H}^{\prime}}\right) \chi^{c}\right\rangle \\
& =\left\langle e^{t \tilde{H}^{\prime}}\right\rangle+\left\langle\chi^{c}\right\rangle-\frac{\left\langle e^{t \tilde{H}^{\prime}} \chi^{c}\right\rangle}{\left\langle\chi^{c}\right\rangle}\left\langle\chi^{c}\right\rangle \leqq\left\langle e^{t \tilde{H}^{\prime}}\right\rangle
\end{aligned}
$$

by Jensen's inequality.

7.3 The Large Fields. Now we discuss the probability distribution of $N_{x}^{k}$, solution of (7.5) and (7.6) and prove Proposition 2. Since $\tilde{H}^{0}$ is Gaussian we have

$$
\operatorname{Prob}\left(N_{x}^{0}=n\right) \leqq \exp \left(-\frac{n^{2} \delta^{2}}{8 \varepsilon^{2}}\right),
$$

and the $n_{x}^{k}$ has a similar bound (7.9), so we expect a Gaussian bound for $N$, which is essentially (7.19) for $N \geqq 1$.

To understand Lemma 2 for $N \leqq 1$, let $n_{x}^{0}=1$, on the first scale, and let all other $n^{k}$ 's be equal to zero. Then (letting, for simplicity, $\alpha=\eta=0$ ) $N_{x}^{k}=L^{-2 k}$ and 
$x$ "falls" into $\mathscr{D}$ when $L^{-k_{0}} \simeq \varepsilon^{2}$. The probability of $n_{x}^{0}=1$ is $\exp \left(-c \delta^{2} / \varepsilon^{2}\right)$, which is indeed $\exp \left(-c^{\prime} \sqrt{N_{x}^{k}} / \varepsilon_{k}^{2}\right)$, with $\varepsilon_{k}^{2}=L^{-k} \varepsilon^{2}$. As mentioned in Sect. 3 , the scale $\left(\varepsilon^{-2}\right.$ here) at which $N_{x}^{k}$ disappears (i.e., $x$ belongs to $\mathscr{D}$ ) is much smaller than the inverse probability of $n_{x}^{0}=1$, which sets the distance scale over which such events occur. In other words, the assumption made above that all $n_{x}^{k}$ but one are zero (up to the scale where the original large field becomes small) was quite probable.

A small problem occurs because, if the exponent in (7.19), (7.20) is not large, then one cannot iterate these bounds. This occurs if $\sqrt{N} \simeq \varepsilon_{k}^{2}=L^{(-1+\eta) k} \varepsilon^{2}$, i.e., $N \simeq L^{(-2+2 \eta) k}$. A priori, we obtain from (7.6) that $N^{k} \in L^{(-2+\alpha) k} \mathbf{N}$, which may indeed be too small. (Remember that $\eta>2 \alpha$ in (7.11); actually, later we shall need $\eta>3 \alpha$.) However, the whole point about the introduction of $\mathscr{D}$ was to remove [see the sum in (7.6)] such small $N$ 's. So, we would like to show that, if $N$ becomes too small, it jumps to zero. This is unfortunately impossible: think of a very long component of $D$ which intersects $L^{k} x$ at only one point for all $k$ (this may happen for some $x^{\prime}$ 's). Then $N_{x}^{k} \simeq L^{(-2+\alpha) k}$, but the component does not necessarily fall into $\mathscr{D}^{k}$. However, as this example indicates, if $N_{x}^{k} \neq 0, N_{y}^{k}$ cannot be small for all $y^{\prime}$ s adjacent to $x$. This motivates the introduction of the following, $\bar{N}_{x}$ variables and is the intuitive reason behind Lemma 1:

$$
\begin{aligned}
& \bar{N}_{x}^{0}=\sum_{|y-x|<2} N_{y}^{0}, \\
& \bar{N}_{x}^{k}=L^{-2+\alpha} \sum_{|y-x|<2} \sum_{z \in L y \backslash \mathscr{D}^{k-1}} \bar{N}_{z}^{k-1}+n_{x}^{k} \text { if } x \in D^{k}, \\
& \bar{N}_{x}^{k}=0 \quad \text { otherwise. }
\end{aligned}
$$

Obviously $\bar{N}_{x}^{k} \geqq N_{x}^{k}$. In fact, we have

Lemma 1. If $N_{x}^{k} \neq 0$, then

$$
\bar{N}_{x}^{k} \geqq L^{-3 k / 2} \varepsilon^{2} .
$$

Lemma 2. There exists a constant $A$, such that, for all $k$ and $x$,

$$
P\left(\bar{N}_{x}^{k}=N\right) \leqq\left\{\begin{array}{ll}
\exp \left(-A N^{2} \delta_{k}^{2} / \varepsilon_{k}^{2}\right) & N \geqq 1 \\
\exp \left(-A \sqrt{N} \delta_{k}^{2} / \varepsilon_{k}^{2}\right) & N>1
\end{array} .\right.
$$

Note that $\bar{N}_{x}^{k}$ may only take values in $L^{-(2-\alpha) k} \mathbf{N}$ and is 0 or $\geqq L^{-3 k / 2} \varepsilon^{2}$.

Moreover, for any connected set $\widetilde{D} \subset D$,

$$
\sum_{x \in \tilde{D}} N_{x}^{k} \geqq c^{k} \sum_{x \in \tilde{D}} \bar{N}_{x}^{k}
$$

where $c$ is a geometrical constant $\left(c^{-1}=\#\{y|y-x|<2\}=27\right)$ independent of $L$. This last inequality is easy to check inductively and implies the bound (6.19).

We prove Lemma 1 inductively in $k . k=0$ is clear. Assume it for $k-1$, and let $N_{x}^{k} \neq 0$. Thus, from (7.6) we get that there is $z \in L x \backslash \mathscr{D}^{k-1}$ such that $N_{z}^{k-1} \neq 0$, or $n_{x}^{k} \neq 0$. The latter case immediately yields $\bar{N}_{x}^{k}>L^{-3 k / 2} \varepsilon^{2}$, so assume $n_{x}^{k}=0$. Considering the definition of $\mathscr{D}$ [starting with (5.10)], we see that $N_{z}^{k-1} \neq 0$, 
$z \in L x \backslash \mathscr{D}^{k-1}$ imply that there is a large component $D_{i}$, of $D^{k-1}$, intersecting $L x$, i.e.,

a) $d\left(D_{i}\right)>L / 4$, or

b) $d\left(D_{i}\right)<L / 4$ but $\left|D_{i}\right|>L^{(1-\alpha) / 2}$, or

c) $d\left(D_{i}\right)<L / 4$ but $\sum_{y \in D_{i}} N_{y}>L L^{-k} \varepsilon^{2}$.

Consider a). Since $D_{i}$ is $L^{1 / 2}$ connected, $d\left(D_{i}\right)>L / 4$ and $D_{i} \cap L x \neq \emptyset$ imply $\left|D_{i} \cap L \bar{x}\right| \geqq c L^{1 / 2}$. By the inductive assumption, $\bar{N}_{y}^{k-1} \geqq L^{-3(k-1) / 2} \varepsilon^{2}$ for all $y \in D_{i}$ and

$$
\bar{N}_{x}^{k} \geqq L^{-(2-\alpha)}\left|D_{i} \cap L \bar{x}\right| L^{-3(k-1) / 2} \varepsilon^{2} \geqq L^{-3 k / 2} \varepsilon^{2}
$$

for $L^{\alpha}$ large. In case (b) we have, $D_{i} \subset L \bar{x}$, and we argue as in a). For (c), again $D_{i} \subset L \bar{x}$ and

$$
\bar{N}_{x}^{k} \geqq L^{-2+\alpha} \sum_{y \in D_{\imath}} N_{y}^{k-1}>L^{-2+\alpha} L L^{-(k-1)} \varepsilon^{2}>L^{-3 k / 2} \varepsilon^{2} .
$$

Proof of Lemma 2. The proof is again inductive in $k . k=0$ is clear, so assume it for $k-1$. (Drop $k-1$ and denote $k$ by prime.) Write

$$
\begin{aligned}
P\left(\bar{N}_{x}^{\prime}=N\right)= & P\left(L^{-2+\alpha} \sum_{z} \bar{N}_{z}=N, n_{x}^{\prime}=0\right) \\
& +P\left(L^{-2+\alpha} \sum_{z} \bar{N}_{z}=N-1, n_{x}^{\prime}=1\right),
\end{aligned}
$$

where $\sum_{z}$ denotes the double sum in (7.16) and the second term is zero if $N-1<0$. Consider first the $n_{x}^{\prime}=0$ term. It is bounded by

$$
P\left(L^{-2+\alpha} \sum \bar{N}_{z}=N\right)=\sum_{\left(N_{z}\right)} P\left(\bar{N}_{z}=N_{z}, \forall z \in L \bar{x}\right)
$$

with sum over $N_{z}$ such that $L^{-2+\alpha} \sum N_{z}=N$. We again use the independence of not-too-near $\bar{N}$ 's: $\bar{N}_{z}$ and $\bar{N}_{z^{\prime}}$, are independent if $\left|z-z^{\prime}\right| \geqq 4$. Thus, let $L \bar{x}=\bigcup_{i=1}^{m^{\prime}} U_{i}$ with points in $U_{i}$ at least of distance four from each other. Again by Schwartz,

$$
\begin{aligned}
P\left(\bar{N}_{z}=N_{z}, \forall z \in L \bar{x}\right) & \leqq \prod_{z \in L \bar{x}} P\left(\bar{N}_{z}=N_{z}\right)^{2-m^{\prime}} \\
& \leqq \exp \left[-2^{-m^{\prime}} A \frac{\delta^{2}}{\varepsilon^{2}}\left(\sum_{z \in L \bar{x}}^{>} N_{z}^{2}+\sum_{z \in L \bar{x}}^{<} \sqrt{N_{z}}\right)\right],
\end{aligned}
$$

with $\sum^{>}\left(\sum^{<}\right)$for $N_{z}>1(\leqq 1)$. We shall shortly prove that for arbitrary positive numbers $N_{z}$ with $L^{-2+\alpha} \sum N_{z}=N$,

$$
\sum^{>} N_{z}^{2}+\sum^{<} \sqrt{N_{z}} \geqq L^{1-3 \alpha}\left\{\begin{array}{ll}
N^{2} & N \geqq 1 \\
\sqrt{N} & N \leqq 1
\end{array} .\right.
$$

Assuming this

Since

$$
(7.24) \leqq \sum_{\left(N_{z}\right)} \exp \left[-2^{-m^{\prime}} A \frac{\delta^{2}}{\varepsilon^{2}} L^{1-3 \alpha}\left\{\begin{array}{c}
N_{2} \\
\sqrt{N}
\end{array}\right\}\right]
$$

$$
\sum_{\left(N_{z}\right)} 1 \leqq\left(L^{(2-\alpha) k} N\right)^{c L^{3}}
$$


(remember that $N_{z} \in L^{(-2+\alpha)(k-1)} \mathbf{N}$ and is less than $L^{2-\alpha} N$ ) we may bound (7.27) by

$$
(7.27) \leqq \exp \left[-4 A \frac{\delta^{\prime 2}}{\varepsilon^{\prime 2}}\left\{\begin{array}{l}
N^{2} \\
\sqrt{N}
\end{array}\right]\right.
$$

with $3 \alpha<\eta, L$ large (as long as $\sqrt{N} \varepsilon^{-2}$ is large enough, which is given by Lemma 1). Equation (7.28) bounds the $n_{x}^{\prime}=0$ term of (7.23). Consider then the $n_{x}^{\prime}=1$ term. It is bounded by

$$
\begin{aligned}
& \min \left(P\left(L^{-2+\alpha} \sum \bar{N}_{z}=N-1\right), P\left(n_{x}^{\prime}=1\right)\right) \\
& \leqq \min \left(P\left(L^{-2+\alpha} \sum \bar{N}_{z}=N-1\right), \exp \left[-\mathcal{O}(1){\delta^{\prime}}^{2} / \varepsilon^{\prime 2}\right]\right)
\end{aligned}
$$

by (7.9). For $N \leqq 3$, the second term in (7.29) is bounded by (7.28) (if $36 A<\mathcal{O}(1)$ ). For $N \geqq 3,(N-1)^{2}>\frac{1}{3} N^{2}$, so apply (7.28) to the first term of (7.29) to bound it by $e^{-(4 / 3) A\left(\delta^{\prime 2} / \varepsilon^{\prime 2}\right) N^{2}}$. Thus the sum (7.23) of $n_{x}^{\prime}=0$ and $n_{x}^{\prime}=1$ terms satisfies (7.19) and (7.20). We are thus left with the proof of (7.26). Let $N \leqq 1$. Since $N_{z}^{2} \geqq \sqrt{N_{z}}$, if $N_{z} \geqq 1$ it suffices to show

$$
\sum_{z \in L \bar{x}} \sqrt{N_{z}} \geqq L^{1-3 \alpha} \sqrt{N}
$$

which follows from $\sum_{z} \sqrt{N_{z}} \geqq \sqrt{\sum_{z} N_{z}}$ and $\sum N_{z}=L^{2-\alpha} N$. Let $N \geqq 1$. By Schwartz

$$
\sum^{>} N_{z}^{2} \geqq \frac{1}{c L^{3}}\left(\sum^{>} N_{z}\right)^{2}
$$

since $\sum_{z} 1 \leqq c L^{3}$. Also

$$
\sum^{<} \sqrt{N_{z}} \geqq \sum^{<} N_{z} \geqq \frac{1}{c L^{3}}\left(\sum^{<} N_{z}\right)^{2},
$$

since $N_{z} \leqq 1$, and thus $\left(1 / c L^{3}\right) \sum^{<} N_{z} \leqq 1$. Combining (7.30) and (7.31),

$$
\begin{aligned}
\sum^{>} N_{z}^{2}+\sum^{<} \sqrt{N_{z}} & \geqq \frac{1}{c L^{3}}\left(\left(\sum^{>} N_{z}\right)^{2}+\left(\sum^{<} N_{z}\right)^{2}\right) \\
& \geqq \frac{1}{2 c L^{3}}\left(\sum N_{z}\right)^{2} \geqq \frac{L^{4-2 \alpha} N^{2}}{2 c L^{3}} \geqq L^{1-3 \alpha} N^{2}
\end{aligned}
$$

for $L$ big.

Given Lemmas 1 and 2, the proof of Proposition 2 is easy. Write first

$$
P\left(N_{x}^{k} \neq 0 \text {, some } k\right) \leqq \sum_{k=0}^{\infty} P\left(N_{x}^{k} \neq 0\right) .
$$

By Lemma 1 ,

$$
P\left(N_{x}^{k} \neq 0\right) \leqq P\left(\bar{N}_{x}^{k} \geqq L^{-3 k / 2} \varepsilon^{2}\right),
$$

and, by Lemma 2,

$$
\begin{aligned}
P\left(\bar{N}_{x}^{k} \geqq L^{-3 k / 2} \varepsilon^{2}\right) \leqq & L^{(2-\alpha) k} \exp \left[-A L^{-3 k / 4} \varepsilon \delta_{k}^{2} \varepsilon_{k}^{-2}\right] \\
& +\sum_{N=1}^{\infty} L^{(2-\alpha) k} \exp \left[-A N^{2} \delta_{k}^{2} \varepsilon_{k}^{-2}\right],
\end{aligned}
$$


where the first term bounds $N \leqq 1$ and $L^{(2-\alpha) k}$ is an upper bound on the number of possible values of $\bar{N}_{x}^{k} \in L^{-(2-\alpha) k} \mathbf{N}$. Now

$$
L^{-3 k / 4} \varepsilon \delta_{k}^{2} \varepsilon_{k}^{-2} \geqq L^{(1 / 4-\eta) k} k^{-2} \varepsilon^{-1} \delta_{0} \geqq L^{k / 5} \varepsilon^{-2}
$$

for $k>c \log \varepsilon^{-1} \equiv k_{0}$ and $\eta$ small. So

$$
\sum_{k=k_{0}}^{\infty} P\left(N_{x}^{k} \neq 0\right) \leqq e^{-1 / \varepsilon^{2}}
$$

while, for $k<k_{0}$, we note that

$$
\begin{aligned}
P\left(N_{x}^{k} \neq 0\right) & \leqq \sum_{l=0}^{k_{0}} \sum_{\left[L^{\left.l-k_{y]}\right]=x}\right.} P\left(n_{y}^{l} \neq 0\right) \leqq \sum_{l=0}^{k_{0}} L^{3(k-l)} \exp \left(-\mathcal{O}(1) \delta_{l}^{2} \varepsilon_{l}^{-2}\right) \\
& \leqq 2 L^{3 k_{0}} \exp \left(-\mathcal{O}(1) \delta^{2} \varepsilon^{-2}\right),
\end{aligned}
$$

but $L^{3 k_{0}}<\varepsilon^{-c}$, and the proof is finished.

\section{Proof of the Main Results}

Here we prove the claims of Sect. 2, starting with a direct proof of the phase transition obtained by applying our induction to $\left\langle\sigma_{x}\right\rangle^{ \pm}$. Then we prove the Peierls' bound, which, of course, yields another proof of the phase transition but gives also via an argument of [27], the exponential decay of the correlation functions.

8.1 The Phase Transition. We will now prove

$$
\overline{\left\langle\sigma_{x}\right\rangle_{V}^{ \pm}}= \pm 1 \mp \mathcal{O}\left(e^{-c / \varepsilon^{2}}+e^{-c \beta}\right)
$$

uniformly in $V$. To show (8.1), we rerun our iteration with a $\sigma$-insertion. Thus consider (take e.g. + b.c.)

$$
m=\frac{1}{Z} \sum e^{-\beta H(\sigma)} \sigma_{x} \equiv \frac{Z_{\sigma}}{Z}
$$

and perform the coarse graining for $Z_{\sigma}$ as for $Z$ in Sect. 4. We need to worry separately about contours surrounding $x$. Suppose first that $x \notin \underline{\Gamma}_{l}$ (the large contours; remember that $\left.D \subset \underline{\Gamma}_{l}\right)$. Consider the component $V_{x}^{ \pm} \subset V^{ \pm}$, where $x$ lies and the small contour sum

$$
Z_{\sigma s}^{ \pm}\left(V_{x}^{ \pm}\right)=\sum_{\Gamma}^{s} \rho(\Gamma) e^{ \pm \beta\left(H, V^{\mp}(\Gamma)\right)} \sigma_{x}(\Gamma)
$$

which we may immediately write, due to the convergence of the small contour expansion, as

$$
Z_{\sigma s}^{ \pm}\left(V_{x}^{ \pm}\right)=Z_{s}^{ \pm}\left(V_{x}^{ \pm}\right)\left( \pm 1+\sum_{C \subset V_{\frac{1}{x}}^{ \pm}} S_{\bar{C}}^{ \pm}\right)
$$

where

$$
\left|S_{\tilde{C}}^{ \pm}\right| \leqq e^{-\tilde{\beta}|C| / 2}
$$

and the $C$ 's contain $x$ and are connected. If $x \in \underline{\Gamma}_{l}$, the contour $\gamma \ni x$ obviously has 
the same upper bound as before. Now block as before, also the $C$ 's of $S_{C}^{ \pm}$, to get

$$
\begin{gathered}
\rho_{\sigma}^{\prime}\left(\gamma^{\prime}\right)=\rho^{\prime}\left(\gamma^{\prime}\right) \quad \text { if } \quad\left[L^{-1} x\right] \notin \gamma^{\prime}, \\
W_{\sigma C}^{\prime}=W_{C}^{\prime} \quad \text { if } \quad\left[L^{-1} x\right] \notin C .
\end{gathered}
$$

By convention, we put $\left[L^{-1} x\right] \in \Gamma^{\prime}$ and for the contours $x_{1}^{ \pm}=\left(\left[L^{-1} x\right], \pm 1\right)$ whose support is reduced to that one point, we have

$$
\rho_{\sigma}^{\prime}\left(x_{1}^{ \pm}\right)= \pm 1+\mathcal{O}\left(e^{-\tilde{\beta}}\right)
$$

if $N_{\left[L^{-1} x\right]}^{\prime}=0$. Otherwise, $\rho_{i}^{\prime}, W_{\sigma}^{\prime}$ have the same upper bounds as $\rho^{\prime}, W^{\prime}$.

Iteration goes exactly as before, only keeping track of contours touching $\left[L^{-k} x\right]$. Consider first the event

$$
A \equiv\left\{N_{\left[L^{-k} x\right]}^{k}=0 \quad \forall k\right\} .
$$

Then (8.6), (8.7) hold for all $k$, with the old bounds except that for $\rho_{\sigma}^{k}(\gamma)$, we have only the upper bound if $\left[L^{-k} x\right] \in \gamma$. Equation (8.8) flows as

$$
\rho_{\sigma}^{k}\left(x_{k}^{ \pm}\right)=\rho_{\sigma}^{k-1}\left(x_{k-1}^{ \pm}\right)+\mathcal{O}\left(e^{-\tilde{\beta}_{k}}\right) \text {, }
$$

i.e., we have, uniformly in $k$,

$$
\rho_{\sigma}^{k}\left(x_{k}^{ \pm}\right)= \pm 1+\mathcal{O}\left(e^{-\tilde{\beta}}\right)
$$

Thus, after $N$ steps (with $V=L^{N}$ ), $V$ is contracted to a point, $\{0\}$ and we have

$$
m=\left(\rho_{\sigma}^{N}\left(0^{+}\right)+e^{-\beta_{N} H^{N}} \rho_{\sigma}^{N}\left(0^{-}\right)\right) Z^{-1},
$$

where in the first term $0^{+}=(0,1)$ and, in the second term, $0^{-}=(0, \sigma) \sigma_{0}=-1$, $\sigma_{x}=1, x \neq 0$ (i.e., outside $V$ ), so

$$
\left|\rho_{\sigma}^{N}\left(0^{-}\right)\right| \leqq e^{-\beta_{N}} .
$$

Since $Z$ is given by $1+\rho^{N}\left(0^{+}\right)+e^{-\beta_{N} H^{N}} \rho^{N}\left(0^{-}\right)$with $\rho^{N}\left(0^{-}\right)$satisfying $(8.10)$ and $\rho^{N}\left(0^{+}\right) \leqq e^{-\tilde{\beta}_{N}}$, we have

$$
m=1-\mathcal{O}\left(e^{-\tilde{\beta}_{0}}\right) .
$$

The event (8.9) has probability

$$
P(A) \geqq 1-e^{-c / \varepsilon^{2}}
$$

by our probability estimates (Prop. 2 of Sect. 7). Thus (8.1) follows, since $|m| \leqq 1$ for all $h$. (On the first scale, $\widetilde{\beta}$ was chosen equal to $\beta^{1 / 2}$, but that can obviously be replaced by $\beta / c(L)$.)

8.2 The Peierls' Bound. One has Peierls' estimate for the usual Ising contours, with, say, + spins outside $\gamma$ :

$$
\operatorname{Pr}(\gamma) \leqq e^{-2 \beta|\gamma|} Z^{-}(\operatorname{Int} \gamma) / Z^{+}(\operatorname{Int} \gamma)
$$

The Peierls' bound holds if

$$
\operatorname{Pr}(\gamma) \leqq e^{-0(\beta) \mid \underline{\underline{\gamma}}}
$$

Let $A_{\gamma}$ be the event corresponding to the Peierls bound i.e., for $h$ in $A_{\gamma}$,

$$
Z^{-}(\operatorname{Int} \gamma) / Z^{+}(\operatorname{Int} \gamma) \leqq e^{\beta \delta|\underline{\underline{y}}|}, \quad \delta \text { small. }
$$


In this section we show, that $A_{\gamma}$, for all $\gamma$ surrounding a fixed site, is likely:

$$
P\left(\exists \gamma \text { with } o \in V(\gamma) \text { and } A_{\gamma} \text { violated }\right) \leqq e^{-c \delta^{2} / \varepsilon^{2}}
$$

(with $P=h$-ensemble). This yields the Peierls bound for all contours surrounding the origin.

The proof of (8.11)-(8.12) consists in running our iteration for the partition function in arbitrary volume $V=\operatorname{Int} \gamma$ and studying the resulting free energy. We write $Z^{ \pm}(\operatorname{Int} \gamma) \equiv Z_{\gamma}^{ \pm}$and perform the RGT as in Sect. 4. Denoting $\left[L^{-1} \gamma\right]$ by $\gamma^{\prime}$, we get as in (4.11),

$$
\log Z_{\gamma}^{-} / Z_{\gamma}^{+}=\beta\left(\sum_{x \in \ln t \gamma} H_{x}+\sum_{C \in \operatorname{Int} \gamma}\left(\delta h_{C}^{-}-\delta h_{C}^{+}\right)\right)+\log Z_{\gamma^{\prime}}^{\prime-} / Z_{\gamma^{\prime}}^{\prime+} .
$$

Note, that $Z_{\gamma^{\prime}}^{\prime \pm}$ are not equal to $Z^{\prime \pm}\left(\operatorname{Int} \gamma^{\prime}\right)$. The former has different activities $\rho^{\prime}\left(\gamma_{\alpha}^{\prime}\right)$ for $\underline{\gamma}_{\alpha}^{\prime}$ touching $\underline{\gamma}^{\prime}$ since contours $\underline{\gamma}_{\beta} \subset$ Int $\gamma$ are blocked to get $\gamma_{\alpha}^{\prime}$, whereas in the latter only $\underline{\gamma}_{\beta} \subset \bar{L}$ Int $\gamma^{\prime}$ enter. However, this fact will only produce boundary terms (i.e., of order $|\underline{\underline{\gamma}}|$ ) in later stages of our induction.

We will write the first term in (8.13) in terms of blocked variables and a boundary correction. Let

$$
H^{\prime}\left(\gamma^{\prime}\right)=\sum_{C \subset \text { Int } \gamma^{\prime}} H_{C}^{\prime}
$$

be the resulting small field term and

$$
R^{\prime}\left(\gamma^{\prime}\right)=\sum_{x \in V\left(\gamma^{\prime}\right)} \tilde{H}_{x}^{\prime} n_{x}^{\prime} \equiv \sum_{x \in V\left(\gamma^{\prime}\right)} r_{x}^{\prime}
$$

the large field term (see (4.26)). Then

$$
\log Z_{\gamma}^{-} / Z_{\gamma}^{+}=\beta^{\prime}\left(H^{\prime}\left(\gamma^{\prime}\right)+R^{\prime}\left(\gamma^{\prime}\right)\right)+F^{\prime}\left(\gamma^{\prime}\right)+\log Z_{\gamma^{\prime}}^{\prime-} / Z_{\gamma^{\prime}}^{\prime+},
$$

where the error $F^{\prime}$ has two sources. First, we have the $H$ not contributing to $H^{\prime}$ or $R^{\prime}$. This is less than

$$
\beta \sum_{x \in L \underline{\underline{\gamma}^{\prime}}}\left|H_{x}\right| \leqq \beta \delta L^{3}|\underline{\gamma}| .
$$

Secondly, we have the $\delta h_{C}^{ \pm}$with $C \cap \underline{\gamma} \neq \emptyset$ that are either overcounted, or absent in (8.14). These are bounded by $e^{-\tilde{\beta} / 2}|\underline{\gamma}|$, so

$$
\left|F^{\prime}\left(\gamma^{\prime}\right)\right| \leqq \beta 0(\delta)|\underline{\gamma}| .
$$

We will now iterate (8.16). At each step we get a sum of $\delta h_{C}^{n+}-\delta h_{C}^{n-}$, with $C \subset \operatorname{Int} \gamma^{n}$, to be added to the previous contributions. We want to distribute them so that a formula like (8.16) iterates.

Since it will be crucial for our argument that we apply probabilistic estimates to $\gamma$-independent random variables, we first define the "infinite volume" free energies to which we will derive $\gamma$-dependent corrections. We denote these variables by boldface, $\mathbf{H}^{n}$ and $\mathbf{N}^{n}$, to distinguish them from the slightly different $H^{n}$ and $N^{n}$.

We define inductively (drop $n$ again)

$$
\tilde{\mathbf{H}}_{x}=L^{-2+\alpha} \sum_{y \in L x} \mathbf{H}_{y}+\sum_{y \in L x \cap \hat{\mathscr{Q}}} \mathbf{r}_{y}+q_{x}
$$


where $q_{x}$ is as in (7.2). The difference between $\tilde{H}$ and $\tilde{\mathbf{H}}$ is that in the latter we reinsert the "small large fields" in $\hat{\mathscr{D}}$ (but they are equal on the first scale). $\mathbf{r}$ is defined inductively by

$$
\mathbf{r}_{x}^{\prime}=L^{-2+\alpha} \sum_{y \in L x \backslash \hat{\mathscr{D}}} \mathbf{r}_{y}+\tilde{\mathbf{H}}_{x} \mathbf{n}_{x}^{\prime}
$$

and, analogously to Sect. 5,

$$
\mathbf{H}_{x}^{\prime}=\tilde{\mathbf{H}}_{x} \chi\left(\left|\tilde{\mathbf{H}}_{x}\right|<\delta^{\prime}\right), \quad \mathbf{n}_{x}^{\prime}=1-\chi\left(\left|\tilde{\mathbf{H}}_{x}\right|<\delta^{\prime}\right) .
$$

Finally, $\mathbf{N}$ is defined in terms of $\mathbf{n}$ as before, and $\hat{\mathscr{D}}$ in terms of $\mathbf{N}$. Let us assume, inductively, that

$$
\log Z_{\gamma}^{-} / Z_{\gamma}^{+}=\beta^{n}\left(\mathbf{H}^{n}\left(\gamma^{n}\right)+\mathbf{R}^{n}\left(\gamma^{n}\right)\right)+F^{n}+\log Z_{\gamma^{\prime}}^{\prime-} / Z_{\gamma^{\prime}}^{\prime+}
$$

where

$$
\mathbf{H}^{n}\left(\gamma^{n}\right)=\sum_{C \subset \operatorname{Int} \gamma^{n}} \mathbf{H}_{C}^{n}
$$

(and $\mathbf{H}_{C}^{n}=H_{C}^{n}$ if $|C|>1$ ),

$$
\mathbf{R}^{n}\left(\gamma^{n}\right)=\sum_{x \in V\left(\gamma^{n}\right)} \mathbf{r}_{x}^{n}(\gamma)
$$

and $F^{n}$ is bounded by $(8.18)$.

The recursion for $\mathbf{r}_{x}^{\prime}(\gamma)$ is as in (8.20), with the crucial difference that $y \in(L x \backslash \hat{\mathscr{D}}) \cap V(\gamma)$. Now note that $\mathbf{r}_{x}^{n}(\gamma)=\mathbf{r}_{x}^{n}$ if $x \in \operatorname{Int} \gamma^{n}$. Moreover $\left|\mathbf{r}_{x}^{n}\right| \leqq \mathbf{N}_{x}^{n}$, and therefore the same is true for $\mathbf{r}_{x}^{n}(\gamma)$ :

$$
\left|\mathbf{r}_{x}^{n}(\gamma)\right| \leqq \mathbf{N}_{x}^{n}
$$

With these definitions we get

$$
\beta(\mathbf{H}(\gamma)+\mathbf{R}(\gamma))+\sum_{C \subset \operatorname{Int} \gamma}\left(\delta h_{C}^{+}-\delta h_{C}^{-}\right)=\beta^{\prime}\left(\mathbf{H}^{\prime}\left(\gamma^{\prime}\right)+\mathbf{R}^{\prime}\left(\gamma^{\prime}\right)\right)+\delta F
$$

In (8.25) we used the fact $L$ Int $\gamma^{n+1} \subset \operatorname{Int} \gamma^{n}$, so that only $\mathbf{r}_{x}^{n}$ (and not $\mathbf{r}_{x}^{n}(\gamma)$ ) enter in the $\mathbf{H}^{n+1}$ on the right-hand side of (8.25). $\delta F$ contains terms coming from $\mathbf{H}(\gamma)$, not included in $\mathbf{H}^{\prime}$, and bounded by

$$
\beta_{n}\left|\partial \operatorname{Int} \gamma^{n}\right| \cdot 0\left(\delta_{n}\right) \leqq 0\left(\delta_{n}\right) \beta|\underline{\gamma}|
$$

a $\delta H_{\bar{C}}^{ \pm}$term, which includes the $\gamma$-dependent contours discussed after Eq. (8.13) and the term

$$
\left|\beta_{n} \sum_{y \in \Delta} \mathbf{r}_{y}^{n}(\gamma)\right| \ll \tilde{\beta}_{n}\left|\gamma^{n}\right|
$$

with $\Delta=\left(L V\left(\gamma^{\prime}\right) \backslash V(\gamma)\right) \cap \hat{\mathscr{D}}$. We used $\left(8.24^{\prime}\right)$ and (5.12) here. Since $|\underline{\gamma}| \geqq(L / 2)^{n}\left|\underline{\gamma}^{n}\right|$ as long as $\left|\underline{\gamma}^{n}\right|>c(L),(8.27)$ may be absorbed in (8.26). Thus $F^{n}$ stays bounded by (8.18) throughout the iteration because $\sum_{n} \delta_{n}<c \delta$. The main point of the proof is that $\mathbf{H}$ and $\mathbf{R}$ are defined in terms of the $\gamma$-independent information $(q)$. The $\gamma$-dependent terms are always controlled by $|\underline{\gamma}|$. We stop the iteration once $\underline{\gamma}^{n}=\{0\}$. Then

$$
\log Z_{\gamma}^{+} / Z_{\gamma}^{-}=\beta^{n} \mathbf{R}^{n}+\log Z_{\gamma^{n}}^{n+} / Z_{\gamma^{n}}^{n-}+0(\delta) \beta|\underline{\gamma}|,
$$


and

$$
\begin{gathered}
\left|\mathbf{R}^{n}\right| \leqq C \mathbf{N}_{0}^{n}, \\
\left|\log Z^{n+} / Z^{n-}\right| \leqq C \beta_{n} N_{0}^{n}+0\left(e^{-\beta_{n}}\right) .
\end{gathered}
$$

Note that the extra term $\mathbf{r}$ in the iteration (8.21), will not affect the analysis of the probability distribution of $\mathbf{N}$ : they have the same bounds as $N$ 's. Thus, the event $\left\{\mathbf{N}_{0}^{n}=0=N_{0}^{n}\right.$ for all $\left.n\right\}$ has the probability $1-\exp \left[-0\left(\delta^{2} / \varepsilon^{2}\right)\right]$, and thus (8.28)(8.30) yield (8.12) (note that it is crucial that $\mathbf{N}$ was defined in a $\gamma$-independent way).

8.3 Exponential Decay of Correlations. To get a short proof, we shall use FKG inequalities (which hold for any realization of the field) following an idea of [27]. A longer, but more robust, proof should be obtained by applying our induction and our proof of the Peierls' bound to a duplicated system.

First of all, by a result of [26], the decay of all correlation functions is dominated by the decay of the two-point function, so we only discuss the latter. By expressing the derivatives in terms of truncated correlation functions, one also obtains that the free energy and the correlation functions are $C^{\infty}$ in $\beta$ and (one-sided) $C^{\infty}$ in $h$, see [26].

Next, we introduce the FKG increasing variables $\rho_{x}=\left(1+\sigma_{x}\right) / 2=0$ or 1 . All we need to prove is

$$
0 \leqq\left\langle\rho_{x} \rho_{y}\right\rangle^{+}-\left\langle\rho_{x}\right\rangle^{+}\left\langle\rho_{y}\right\rangle^{+} \leqq c(h) \exp (-m|x-y|),
$$

where $c(h)<\infty$ a.e. The first inequality is FKG. An upper bound on $\left\langle\rho_{x} \rho_{y}\right\rangle_{V}^{+}$is $\left\langle\rho_{x}\right\rangle_{V_{1}}^{+}\left\langle\rho_{y}\right\rangle_{V_{2}}^{+}$, where $x \in V_{1}, y \in V_{2}$ and $V_{1}, V_{2}$ are obtained by putting a "wall" of + spins through $V$, halfway between $x$ and $y$. Thus we condition on an increasing event, and this gives an upper bound, by FKG. Therefore (8.31) holds if we prove that $\left\langle\rho_{0}\right\rangle_{V}^{+}$attains its thermodynamic limit exponentially fast:

$$
0 \leqq\left\langle\rho_{0}\right\rangle_{V}^{+}-\left\langle\rho_{0}\right\rangle_{V^{\prime}}^{+} \leqq c(h) \exp \left(-m d\left(0, V^{c}\right)\right)
$$

for $0 \in V \subset V^{\prime}$. The first inequality is again FKG. Now let $E$ be the event: there is no contour in $V^{\prime}$ which contains, in its interior, 0 and some point of $\partial V$. Now, it follows from FKG and a simple geometrical observation (see [27]) that

$$
\left\langle\rho_{0} \mid E\right\rangle_{V^{\prime}}^{+} \leqq\left\langle\rho_{0}\right\rangle_{V}
$$

because conditioning on $E$ means that we may find a region $\tilde{V} \subset V$, with $x \in \tilde{V}$, on the boundary of which the spins are +1 . Equation (8.33) follows then from the monotonicity in the volume. Thus, to get (8.31), we have to bound $\operatorname{Pr}\left(E^{c}\right)$ by the right-hand side of (8.31). It is clear that, if the Peierls' bound holds for all contours enclosing $x$,

$$
\operatorname{Pr}\left(E^{c}\right) \leqq \sum \operatorname{Pr}(\gamma) \leqq \exp \left(-m d\left(0, V^{c}\right)\right),
$$

where the sum is over $\gamma$ with $0 \in \operatorname{Int} \gamma$, Int $\gamma \cap \partial V \neq \emptyset$ with $m=0(\beta)$. However, it is also clear that, for (8.34) to hold we only need the Peierls' bound for "long" contours $\left(d(\gamma) \geqq d\left(0, V^{c}\right)\right)$. Going back to Sect. 8.2, we see that this holds provided $N_{x}^{k}=0$ (and $\left.\mathbf{N}_{x}^{k}=0\right)$ all $|x|<L$ and all $k>(\log L)^{-1} \log d\left(x, V^{c}\right)$ (this is the scale where contours contributing to (8.34) become small). 
So, we may write

$$
\operatorname{Pr}\left(E^{c}\right) \leqq c(h) \exp \left(-m d\left(x, V^{c}\right)\right)
$$

with $c(h)=\exp m L^{k}$ and $k=k(h)=\max \left\{k|\exists x| x \mid, \leqq L, N_{x}^{k}\right.$ or $\left.\mathbf{N}_{x}^{k} \neq 0\right\}$. Using the results of Sect. 7.3 one sees that $c(h)<\infty$ with probability one.

Acknowledgements. It is a pleasure to thank B. Derrida, D. Fisher, G. Grinstein, J. Imbrie, J. Wehr and especially T. Spencer for interesting discussions and comments. J.B. would like to thank J. Lebowitz at Rutgers University for hospitality as well as the Research Institute for Theoretical Physics at Helsinki University. A.K. would like to thank The Institute for Advanced Study for hospitality. This work was supported in part by NSF Grant No. DMR86-12369 and by NSF Grant No. PHY82-17853, supplemented by funds from NASA.

\section{References}

1. Fishman, S., Aharony, A.: Random Field effects in disordered anisotropic antiferromagnets. J. Phys. C 12, L729-733 (1976)

2. Villain, J.: Commensurate-incommensurate transition with frozen impurities. J. Phys. 43, L551-558 (1982)

3. Grinstein, G., Fernandez, J.: Equilibration in random-field Ising systems. Phys. Rev. B 29, 6389-6392 (1984)

4. Pytte, E., Imry, Y., Mukamel, D.: Lower critical dimension and the roughening transition of the random-field Ising model. Phys. Rev. Lett. 46, 1173-1177 (1981)

5. Mukamel, D., Pytte, E.: Interface fluctuations and the Ising model in a random field. Phys. Rev. B 25, 4779-4786 (1982)

6. Grinstein, G.: Ferromagnet phase transitions in random fields: The breakdown of scaling laws. Phys. Rev. Lett. 37, 944-947 (1976)

7. Aharony, A., Imry, Y., Ma, S. K.: Lowering of dimensionality in phase transitions with random fields. Phys. Rev. Lett. 37, 1364-1367 (1976)

8. Young, A. P.: On the lowering of dimensionality in phase transitions with random fields. J. Phys. C 10, L257-262 (1977)

9. Parisi, G., Sourlas, N.: Random magnetic fields, supersymmetry, and negative dimensions. Phys. Rev. Lett. 43, 744-745 (1979); Supersymmetric field theories and stochastic differential equations. Nucl. Phys. B 206, 321-332 (1982)

10. Kogon, H. S., Wallace, D. J.: The Ising model in a random field; supersymmetric surface fluctuations and their implications in three dimensions. J. Phys. A 14, L527-531 (1981)

11. Klein, A., Landau, L., Perez, J. F.: Supersymmetry and the Parisi-Sourlas dimensional reduction: A rigorous proof. Commun. Math. Phys. 93, 459-482 (1984)

12. Parisi, G.: An introduction to the statistical mechanics of amorphous systems. In: Recent advances in field theory and statistical mechanics, Zuber, J. B., Stora, R. (eds.) Amsterdam: North-Holland 1984

13. Fisher, D.: Interface fluctuations in disordered systems: $5-\varepsilon$ expansion and failure of dimensional reduction. Phys. Rev. Lett. 56, 1964-1967 (1986)

14. Imry, Y., Ma, S. K.: Random-field instability of the ordered state of continuous symmetry. Phys. Rev. Lett. 35, 1399-1401 (1975)

15. Grinstein, G., Ma, S. K.: Roughening and lower critical dimension in the random-field Ising model. Phys. Rev. Lett. 49, 685-88 (1982)

16. Fisher, D., Fröhlich, J., Spencer, T.: The Ising model in a random magnetic field. J. Stat. Phys. 34, 863-70 (1984)

17. Chalker, J.: On the lower critical dimensionality of the Ising model in a random field. J. Phys. C 16, 6615-6622 (1983)

18. Imbrie, J.: The ground state of the three-dimensional random-field Ising model. Commun. Math. Phys. 98, 145-176 (1985) 
19. Beretti, A.: Some properties of random Ising models. J. Stat. Phys. 38, 483 (1985)

20. Fröhlich, J., Imbrie, J.: Improved perturbation expansion for disordered systems: Beating Griffiths' singularities. Commun. Math Phys. 96, 145-180 (1984)

21. The hierarchical random field Ising model. Preprint.

22. Derrida, B., Shnidman, Y.: Possible line of critical points for a random field Ising model in dimension 2. J. Phys. Lett. 45, L577-L581 (1984)

23. Bricmont, J., Kupiainen, A.: Lower critical dimension for the random field Ising model. Phys. Rev. Lett. 59, 1829-1832 (1987)

24. Gawedzki, K., Kotecky, R., Kupiainen, A.: Coarse graining approach to first order phase transitions. J. Stat. Phys. (1987)

25. Griffiths, R. B.: Nonanalytic behavior above the critical point in a random Ising ferromagnet. Phys. Rev. Lett. 23, 17 (1969)

26. Lebowitz, J. L.: Bounds on the correlations and analyticity properties of ferromagnetic Ising spin systems. Commun. Math. Phys. 32, 313-322 (1973)

27. Martin-Löf, A.: Mixing properties, differentiability of the free energy and central limit theorem for a pure phase in the Ising model at low temperature. Commun. Math. Phys. 32, 75-92 (1973)

Communicated by T. Spencer

Received September 1, 1987 